\title{
The SUSY seesaw model and lepton-flavor violation at a future electron-positron linear collider
}

\author{
F. Deppisch ${ }^{1,2 *}$, H. Päs ${ }^{1 \dagger}$, A. Redelbach ${ }^{1 \ddagger}$, R. Rückl ${ }^{1 \S}$, Y. Shimizu ${ }^{3 థ}$ \\ ${ }^{1}$ Institut für Theoretische Physik und Astrophysik \\ Universität Würzburg \\ D-97074 Würzburg, Germany \\ ${ }^{2}$ Institut de Física Corpuscular - C.S.I.C., Universitat de València \\ Edifici Instituts d'Investigació - Apartat de Correus 22085 - 46071 València, Spain \\ 3 Department of Physics \\ Nagoya University \\ Nagoya, 464-8602, Japan
}

\begin{abstract}
We study lepton-flavor violating slepton production and decay at a future $e^{+} e^{-}$ linear collider in context with the seesaw mechanism in mSUGRA post-LEP benchmark scenarios. The present knowledge in the neutrino sector as well as improved future measurements are taken into account. We calculate the signal cross-sections $\sigma\left(e^{ \pm} e^{-} \rightarrow l_{\beta}^{ \pm} l_{\alpha}^{-} \tilde{\chi}_{b}^{0} \tilde{\chi}_{a}^{0}\right) ; l_{\delta}=e, \mu, \tau ; \alpha \neq \beta$ and estimate the main background processes. Furthermore, we investigate the correlations of these signals with the corresponding lepton-flavor violating rare decays $l_{\alpha} \rightarrow l_{\beta} \gamma$. It is shown that these correlations are relatively weakly affected by uncertainties in the neutrino data, but very sensitive to the model parameters. Hence, they are particularly suited for probing the origin of lepton-flavor violation.
\end{abstract}

\footnotetext{
*E-mail: deppisch@physik.uni-wuerzburg.de

$\dagger$ E-mail: paes@physik.uni-wuerzburg.de

${ }^{\ddagger}$ E-mail: asredelb@physik.uni-wuerzburg.de

§E-mail: rueckl@physik.uni-wuerzburg.de

『E-mail: shimizu@eken.phys.nagoya-u.ac.jp
} 


\section{Introduction}

One of the main virtues of experiments at an $e^{+} e^{-}$linear collider (LC) is the clean environment allowing studies of the production and decay of new particles with low background. This not only enables precision measurements of particle properties, but also searches for very rare processes and small effects. An important example of this kind is lepton-flavor violation (LFV) as suggested by the experimental evidence for neutrino oscillations [1, 2] and expected particularly in SUSY models. Phenomenological investigations have indicated how tests of LFV at a high-energy LC could nicely complement searches for lepton-flavor violating rare decays such as $\mu \rightarrow e \gamma$. Previous work [3] has mainly focussed on slepton-pair production assuming two-generation slepton mixing. LFV in chargino-pair production was analyzed in [4] together with a detailed background analysis. Recently, it was shown in a rather model-independent analysis [5] by scanning over all possible soft SUSY-breaking terms consistent with the existing low-energy bounds that LFV in the above processes may be quite sizable, leading to final states with a pair of charged leptons of unequal flavor, missing energy, and possibly additional leptons and jets.

In supersymmetric theories with heavy right-handed Majorana neutrinos, the seesaw mechanism [6] can give rise to light neutrino masses at or below the sub-eV scale. Moreover, the massive neutrinos affect the renormalization group running of the slepton masses, which leads to mixing of different slepton flavors. In the present article we investigate the implications of recent neutrino measurements on this mixing, extending our previous analysis of the radiative decays $l_{\alpha} \rightarrow l_{\beta} \gamma$ in the SUSY seesaw model [7] to the lepton-flavor violating processes $e^{ \pm} e^{-} \rightarrow l_{\beta}^{ \pm} l_{\alpha}^{-} \tilde{\chi}_{b}^{0} \tilde{\chi}_{a}^{0}$ involving slepton-pair production and subsequent decay. We again use the mSUGRA benchmark scenarios proposed in [8] for LC studies, concentrating on those which predict charged sleptons that are light enough to be pair-produced at the center-of-mass energy $\sqrt{s}=500 \mathrm{GeV}$. Furthermore, we examine the most important background processes and indicate the most promising lepton-flavor violating channels. The fact that in the benchmark models considered LFV occurs only in the left-handed slepton sector makes the separation of signal and background more difficult than in models with LFV in the right-handed sector. Therefore, our choice of models provides good study cases for developing search strategies. Finally, we work out the correlations between LFV in the high-energy $e^{ \pm} e^{-}$collisions and the radiative lepton decays, and show that these correlations are relatively weakly affected by the uncertainties in the neutrino data, but very sensitive to the mSUGRA parameters. Consequently, they could play an important role in probing the class of models of LFV studied here. 
The paper is organized as follows. In section 2 we briefly outline the SUSY seesaw mechanism and discuss the slepton and neutralino mass matrices. Section 3 summarizes our analytic results on the helicity amplitudes for slepton-pair production, $e^{ \pm} e^{-} \rightarrow \tilde{l}_{j}^{ \pm} \tilde{l}_{i}^{-}$, and slepton decay, $\tilde{l}_{i}^{ \pm} \rightarrow l_{\alpha}^{ \pm} \tilde{\chi}_{a}^{0}$, and provides the cross-section formula for the complete $2 \rightarrow 4$ processes $e^{ \pm} e^{-} \rightarrow l_{\beta}^{ \pm} l_{\alpha}^{-} \tilde{\chi}_{b}^{0} \tilde{\chi}_{a}^{0}$. The fundamental parameters of the mSUGRA benchmark scenarios as well as the neutrino data used in our numerical analysis are specified in section 4 . In this section we also present our predictions on the signal cross-sections, while the estimates of the background and the effects of energy and angular cuts are given in section 5 . The prospects for LC searches anticipated on the basis of our analysis are summarized in section 6 .

\section{Lepton-flavor violation in the charged slepton sector}

\subsection{Supersymmetric seesaw mechanism}

In supersymmetric models the existence of right-handed neutrino singlet fields with Majorana mass terms and Yukawa interactions gives rise to the well-known seesaw mechanism, provided the Majorana mass scale $M_{R}$ is much higher than the scale $v=174 \mathrm{GeV}$ of electroweak symmetry breaking. At energies below $M_{R}$ the relevant term in the effective superpotential is given by 9

$$
W_{\nu}^{e f f}=\frac{1}{2}\left(Y_{\nu} L \cdot H_{2}\right)^{T} M^{-1}\left(Y_{\nu} L \cdot H_{2}\right),
$$

where $L$ denotes the left-handed lepton doublets, $H_{2}$ the Higgs doublet with hypercharge $+\frac{1}{2}$, and $Y_{\nu}$ the matrix of neutrino Yukawa couplings. After electroweak symmetry breaking, the Yukawa couplings generate the Dirac mass matrix $m_{D}=Y_{\nu}\left\langle H_{2}^{0}\right\rangle,\left\langle H_{2}^{0}\right\rangle=v \sin \beta$ being the $H_{2}$ vacuum expectation value with $\tan \beta=\frac{\left\langle H_{2}^{0}\right\rangle}{\left\langle H_{1}^{0}\right\rangle}$. This in turn leads to the Majorana mass matrix

$$
M_{\nu}=m_{D}^{T} M^{-1} m_{D}=Y_{\nu}^{T} M^{-1} Y_{\nu}(v \sin \beta)^{2}
$$

for the light neutrinos which is diagonalized by the unitary MNS matrix $U$ :

$$
U^{T} M_{\nu} U=\operatorname{diag}\left(m_{1}, m_{2}, m_{3}\right) .
$$

The matrix $U$ and the mass eigenvalues $m_{i}$ are constrained by neutrino data as discussed, e.g., in [7]. 


\subsection{Renormalization group evolution of the slepton masses}

The heavy neutrino mass eigenstates contribute to the renormalization group running of the slepton mass matrices thereby inducing flavor non-diagonal terms which are responsible for the lepton-flavor violating processes described later. At the unification scale $M_{X}$ we assume the mSUGRA universality conditions

$$
m_{\tilde{l}_{L}}^{2}=m_{0}^{2} \mathbf{1}, \quad m_{\tilde{l}_{R}}^{2}=m_{0}^{2} \mathbf{1}, \quad A=A_{0} Y_{l},
$$

where $m_{0}$ is the common scalar mass and $A_{0}$ the common trilinear coupling. At lower scales of order of the SUSY threshold, the mass squared matrix of the charged sleptons has the form

$$
m_{\tilde{l}}^{2}=\left(\begin{array}{cc}
m_{\tilde{l}_{L}}^{2} & m_{\tilde{l}_{L R}}^{2 \dagger} \\
m_{\tilde{l}_{L R}}^{2} & m_{\tilde{l}_{R}}^{2}
\end{array}\right),
$$

where $m_{\tilde{l}_{L}}^{2}, m_{\tilde{l}_{R}}^{2}$ and $m_{\tilde{L}_{L R}}^{2}$ are $3 \times 3$ matrices in flavor space, $m_{\tilde{l}_{L}}^{2}$ and $m_{\tilde{l}_{R}}^{2}$ being hermitian. The respective matrix elements are given by

$$
\begin{aligned}
\left(m_{\tilde{l}_{L}}^{2}\right)_{i j} & =\left(m_{L}^{2}\right)_{i j}+\delta_{i j}\left(m_{l_{i}}^{2}+m_{Z}^{2} \cos 2 \beta\left(-\frac{1}{2}+\sin ^{2} \theta_{W}\right)\right) \\
\left(m_{\tilde{l}_{R}}^{2}\right)_{i j} & =\left(m_{R}^{2}\right)_{i j}+\delta_{i j}\left(m_{l_{i}}^{2}-m_{Z}^{2} \cos 2 \beta \sin ^{2} \theta_{W}\right) \\
\left(m_{\tilde{l}_{L R}}^{2}\right)_{i j} & =A_{i j} v \cos \beta-\delta_{i j} m_{l_{i}} \mu \tan \beta,
\end{aligned}
$$

$\theta_{W}$ being the weak-mixing angle, and $\mu$ the SUSY Higgs-mixing parameter. The contributions to the first terms on the r.h.s. of (6) - (18) are indicated below:

$$
\begin{aligned}
m_{L}^{2} & =m_{0}^{2} \mathbf{1}+\left(\delta m_{L}^{2}\right)_{\mathrm{MSSM}}+\delta m_{L}^{2} \\
m_{R}^{2} & =m_{0}^{2} \mathbf{1}+\left(\delta m_{R}^{2}\right)_{\mathrm{MSSM}}+\delta m_{R}^{2} \\
A & =A_{0} Y_{l}+\delta A_{\mathrm{MSSM}}+\delta A .
\end{aligned}
$$

Here, $\left(\delta m_{L, R}^{2}\right)_{\text {MSSM }}$ and $\delta A_{\text {MSSM }}$ denote the usual MSSM renormalization group corrections [10] which are flavor-diagonal. In addition, the right-handed neutrinos radiatively induce the flavor off-diagonal terms $\delta m_{L, R}$ and $\delta A$. In leading logarithmic approximation ${ }^{1}$, one gets [13]

$$
\delta m_{L}^{2}=-\frac{1}{8 \pi^{2}}\left(3 m_{0}^{2}+A_{0}^{2}\right)\left(Y_{\nu}^{\dagger} L Y_{\nu}\right)
$$

\footnotetext{
${ }^{1}$ The exact one-loop renormalization group equations may give somewhat different results as pointed out in [11] and studied in more detail for lepton-flavor violating processes of interest in a forthcoming article [12. For consistency with the results on radiative decays, derived in [7] and used in the present analysis, we restrict ourselves here to the approximation (12)-(14).
} 


$$
\begin{aligned}
\delta m_{R}^{2} & =0 \\
\delta A & =-\frac{3 A_{0}}{16 \pi^{2}}\left(Y_{l} Y_{\nu}^{\dagger} L Y_{\nu}\right)
\end{aligned}
$$

with

$$
L_{i j}=\ln \left(\frac{M_{X}}{M_{i}}\right) \delta_{i j}
$$

$M_{i}, i=1,2,3$ being the eigenvalues of the Majorana mass matrix $M$ which may be chosen diagonal. Using (2) and (3), the Yukawa matrix $Y_{\nu}$ can be parametrized as follows [9]:

$$
Y_{\nu}=\frac{1}{v \sin \beta} \operatorname{diag}\left(\sqrt{M_{1}}, \sqrt{M_{2}}, \sqrt{M_{3}}\right) R \operatorname{diag}\left(\sqrt{m_{1}}, \sqrt{m_{2}}, \sqrt{m_{3}}\right) U^{\dagger}
$$

where $R$ is an undetermined complex orthogonal matrix. For substitution in (12) and (14) the Yukawa couplings have to be evolved from the low-energy scale taken to be $M_{Z}$ to the Majorana scale $M_{R}$ and further to the GUT scale $M_{X}$.

Following [7] and many previous studies, we assume degenerate Majorana masses $M_{1,2,3}=$ $M_{R}$ and take $R$ to be real. In this case, $R$ drops out and the product $Y_{\nu}^{\dagger} L Y_{\nu}$ is simply given by

$$
Y_{\nu}^{\dagger} L Y_{\nu}=Y_{\nu}^{\dagger} Y_{\nu} \ln \left(\frac{M_{X}}{M_{R}}\right)=\frac{M_{R}}{v^{2} \sin ^{2} \beta} U \cdot \operatorname{diag}\left(m_{1}, m_{2}, m_{3}\right) \cdot U^{\dagger} \ln \left(\frac{M_{X}}{M_{R}}\right) .
$$

A more general investigation allowing for non-degenerate Majorana masses is in progress and will be reported elsewhere [14. Here, we stick to (17) since we want to investigate the correlations between the high-energy processes, which are the main subject of the present paper, and the low-energy rare decays studied in [7]. There, one can find some preliminary qualitative remarks on the effects of a complex matrix $R$.

From (15) the mass eigenvalues $m_{\tilde{l}_{i}}, i=1, \ldots, 6$ of the sleptons are obtained via diagonalization by a $6 \times 6$ unitary matrix $U_{\tilde{l}}$ :

$$
U_{\tilde{l}}^{\dagger} m_{\tilde{l}}^{2} U_{\tilde{l}}=\operatorname{diag}\left(m_{\tilde{l}_{1}}^{2}, \ldots, m_{\tilde{l}_{i}}^{2}, \ldots, m_{\tilde{l}_{6}}^{2}\right) .
$$

For definiteness, the eigenvalues are ordered such that the masses increase from $\tilde{l}_{1}$ to $\tilde{l}_{6}$. In other words, in the absense of LFV $\tilde{l}_{1}=\tilde{\tau}_{1}, \tilde{l}_{2}=\tilde{\mu}_{1}, \ldots, \tilde{l}_{6}=\tilde{\tau}_{2}$. The corresponding mass eigenstates are then expressed in terms of the gauge eigenstates by

$$
\tilde{l}_{i}=\left(U_{\tilde{l}}^{*}\right)_{\alpha i} \tilde{l}_{L \alpha}+\left(U_{\tilde{l}}^{*}\right)_{(\alpha+3) i} \tilde{l}_{R \alpha}, \quad i=1, \ldots, 6 ; \alpha=e, \mu, \tau .
$$

\subsection{Neutralino mass matrix}

In slepton production and decay neutralinos play an important role. Therefore, we want to clarify our notation for later use. The physical neutralinos are mixtures of gauginos and 
higgsinos. In the gauge-eigenstate basis $\psi^{0}=\left(\tilde{B}, \tilde{W}^{0}, \tilde{H}_{1}^{0}, \tilde{H}_{2}^{0}\right)^{T}$, the neutralino mass term reads 15 ]

$$
\mathcal{L}_{\psi}=-\frac{1}{2}\left(\psi^{0}\right)^{T} M_{\psi} \psi^{0}+\text { h.c. }
$$

with

$$
M_{\psi}=\left(\begin{array}{cccc}
M_{1} & 0 & -m_{Z} s_{W} c_{\beta} & m_{Z} s_{W} s_{\beta} \\
0 & M_{2} & m_{Z} c_{W} c_{\beta} & -m_{Z} c_{W} s_{\beta} \\
-m_{Z} s_{W} c_{\beta} & m_{Z} c_{W} c_{\beta} & 0 & -\mu \\
m_{Z} s_{W} s_{\beta} & -m_{Z} c_{W} s_{\beta} & -\mu & 0
\end{array}\right),
$$

$M_{1}$ being the $U(1)$ and $M_{2}$ the $S U(2)$ gaugino mass. The abbreviations $s_{\phi}=\sin \phi$ and $c_{\phi}=\cos \phi$ introduced above will be used throughout the paper.

The neutralino mass eigenstates are given by

$$
\chi_{a}^{0}=N_{a b} \psi_{b}^{0}, \quad a, b=1, \ldots, 4,
$$

where $N$ is the unitary matrix diagonalizing $M_{\psi}$ :

$$
N^{*} M_{\psi} N^{-1}=\operatorname{diag}\left(m_{\tilde{\chi}_{1}^{0}}, m_{\tilde{\chi}_{2}^{0}}, m_{\tilde{\chi}_{3}^{0}}, m_{\tilde{\chi}_{4}^{0}}\right)
$$

and $m_{\tilde{\chi}_{a}^{0}}, a=1, \ldots, 4$ are the mass eigenvalues. Finally, the Majorana spinors for the neutralinos are composed of the Weyl spinors (22) as follows:

$$
\tilde{\chi}_{a}^{0}=\left(\begin{array}{c}
\chi_{a}^{0} \\
\bar{\chi}_{a}^{0}
\end{array}\right) .
$$

\section{Amplitudes and cross-sections}

The flavor mixing in the slepton sector induced by the heavy neutrinos as outlined in section 2.2 gives rise to the lepton-flavor violating processes $e^{ \pm} e^{-} \rightarrow \tilde{l}_{j}^{ \pm} \tilde{l}_{i}^{-} \rightarrow l_{\beta}^{ \pm} l_{\alpha}^{-} \tilde{\chi}_{b}^{0} \tilde{\chi}_{a}^{0}$, $i, j=1, \ldots, 6$. More specifically, in these processes LFV is caused by the slepton mixing matrix $U_{\tilde{l}}$ in (19), which enters both the slepton production and the decay vertices. As a consequence, factorization in production cross-section times branching ratios is not appropriate. One rather has to coherently sum over the intermediate slepton states. In the following, we summarize our analytical results on the amplitudes and cross-sections for the above processes. The detailed calculations have been carried out in [16] and [17]. 


\subsection{The lepton-flavor violating vertices}

The lowest-order Feynman diagrams for the processes under consideration are shown in Fig. 1 and Fig. 2 with the particle four-momenta being defined in brackets. While the $\gamma \tilde{l}_{i}^{+} \tilde{l}_{i}^{-}$vertex appearing only in Fig. 1 is flavor-diagonal, LFV occurs in both the $Z \tilde{l}_{j}^{+} \tilde{l}_{i}^{-}$and $l_{\alpha}^{-} \tilde{l}_{i}^{-} \tilde{\chi}_{a}^{0}$ vertices described by

$$
-i \frac{e}{c_{W} s_{W}} z_{i j}\left(p_{3}-p_{4}\right)^{\mu}
$$

with

$$
z_{i j}=-\frac{1}{2} \sum_{\alpha=1}^{3}\left(U_{\tilde{l}}\right)_{i \alpha}\left(U_{\tilde{l}}^{*}\right)_{j \alpha}+s_{W}^{2} \delta_{i j}
$$

and

$$
-\sqrt{2} i e\left(A_{i \alpha, a} P_{L}+B_{i \alpha, a} P_{R}\right)
$$

with

$$
\begin{aligned}
A_{i \alpha, a} & =\frac{m_{\alpha} N_{a 3}^{*}}{2 m_{W} s_{W} c_{\beta}}\left(U_{\tilde{l}}\right)_{i(\alpha+3)}-\frac{N_{a 1}^{*} t_{W}+N_{a 2}^{*}}{2 s_{W}}\left(U_{\tilde{l}}\right)_{i \alpha} \\
B_{i \alpha, a} & =\frac{N_{a 1}}{c_{W}}\left(U_{\tilde{l}}\right)_{i(\alpha+3)}+\frac{m_{\alpha} N_{a 3}}{2 m_{W} s_{W} c_{\beta}}\left(U_{\tilde{l}}\right)_{i \alpha},
\end{aligned}
$$

respectively. Here, $P_{L, R}=\frac{1}{2}\left(1 \mp \gamma_{5}\right)$ are the left-right projectors, while the matrix elements $N_{a b}$ are defined in (22), and $U_{i \alpha}$ in (19). The $l_{\beta}^{+} \tilde{l}_{j}^{+} \tilde{\chi}_{b}^{0}$ vertex is obtained from (27) after replacing $A_{i \alpha, a}$ by $B_{j \beta, b}^{*}$ and $B_{i \alpha, a}$ by $A_{j \beta, b}^{*}$.

\subsection{Helicity amplitudes for slepton production and decay}

Although we will not consider $e^{ \pm}$-beam polarization in the subsequent numerical analysis, we nevertheless present here the helicity amplitudes for future studies of polarization effects. In comparison to the slepton and neutralino masses, and to the cms energy of a LC the masses of the initial and final state leptons can be neglected in the kinematics. In terms of the Mandelstam variables $s=\left(p_{1}+p_{2}\right)^{2}, t=\left(p_{1}-p_{3}\right)^{2}, u=\left(p_{1}-p_{4}\right)^{2}$ the helicity amplitudes $M_{i j}\left(h_{e^{-}}, h_{e^{+}}\right)(h= \pm 1)$ for the production process $e^{+} e^{-} \rightarrow \tilde{l}_{j}^{+} \tilde{l}_{i}^{-}$are then given by

$$
\begin{aligned}
& M_{i j}(+,+)=-2 i e^{2} \sqrt{s} \sum_{c=1}^{4} m_{\tilde{\chi}_{c}^{0}} \frac{B_{i 1, c} A_{j 1, c}^{*}}{t-m_{\tilde{\chi}_{c}^{0}}^{2}} \\
& M_{i j}(-,-)=-2 i e^{2} \sqrt{s} \sum_{c=1}^{4} m_{\tilde{\chi}_{c}^{0}} \frac{A_{i 1, c} B_{j 1, c}^{*}}{t-m_{\tilde{\chi}_{c}^{0}}^{2}} \\
& M_{i j}(+,-)=-2 i e^{2} \sqrt{t u-p_{3}^{2} p_{4}^{2}}\left(\frac{\delta_{i j}}{s}+\frac{1}{c_{W}^{2}} \frac{z_{i j}}{s-m_{Z}^{2}}+\sum_{c=1}^{4} \frac{B_{i 1, c} B_{j 1, c}^{*}}{t-m_{\tilde{\chi}_{c}^{0}}^{2}}\right)
\end{aligned}
$$




$$
M_{i j}(-,+)=+2 i e^{2} \sqrt{t u-p_{3}^{2} p_{4}^{2}}\left(\frac{\delta_{i j}}{s}+\frac{s_{W}^{2}-\frac{1}{2}}{s_{W}^{2} c_{W}^{2}} \frac{z_{i j}}{s-m_{Z}^{2}}+\sum_{c=1}^{4} \frac{A_{i 1, c} A_{j 1, c}^{*}}{t-m_{\tilde{\chi}_{c}^{0}}^{2}}\right) .
$$

Analogously, the helicity amplitudes $M_{i j}\left(h_{e^{-}}, h_{e^{-}}\right)$for $e^{-} e^{-} \rightarrow \tilde{l}_{i}^{-} \tilde{l}_{j}^{-}$read:

$$
\begin{aligned}
& M_{i j}(+,+)=2 i e^{2} \sqrt{s} \sum_{c=1}^{4} m_{\tilde{\chi}_{c}^{0}} \frac{B_{i 1, c} B_{j 1, c}}{t-m_{\tilde{\chi}_{c}^{0}}^{2}}+(t \rightarrow u, i \leftrightarrow j) \\
& M_{i j}(-,-)=2 i e^{2} \sqrt{s} \sum_{c=1}^{4} m_{\tilde{\chi}_{c}^{0}} \frac{A_{i 1, c} A_{j 1, c}}{t-m_{\tilde{\chi}_{c}^{0}}^{2}}+(t \rightarrow u, i \leftrightarrow j) \\
& M_{i j}(+,-)=2 i e^{2} \sqrt{t u-p_{3}^{2} p_{4}^{2}} \sum_{c=1}^{4} \frac{B_{i 1, c} A_{j 1, c}}{t-m_{\tilde{\chi}_{c}^{0}}^{2}}-(t \rightarrow u, i \leftrightarrow j) \\
& M_{i j}(-,+)=-2 i e^{2} \sqrt{t u-p_{3}^{2} p_{4}^{2}} \sum_{c=1}^{4} \frac{A_{i 1, c} B_{j 1, c}}{t-m_{\tilde{\chi}_{c}^{0}}^{2}}-(t \rightarrow u, i \leftrightarrow j) .
\end{aligned}
$$

Furthermore, for the decay $\tilde{l}_{i}^{-} \rightarrow l_{\alpha}^{-} \tilde{\chi}_{a}^{0}$ the helicity amplitudes $M_{i}^{-}\left(h_{l_{\alpha}^{-}}\right)$summed over the helicity of the neutralino are given by

$$
\begin{aligned}
& M_{i}^{-}(+)=2 i e B_{i \alpha, a}^{*} \sqrt{p_{\alpha} \cdot p_{a}} \\
& M_{i}^{-}(-)=2 i e A_{i \alpha, a}^{*} \sqrt{p_{\alpha} \cdot p_{a}}
\end{aligned}
$$

From these one can obtain the helicity amplitudes $M_{j}^{+}\left(h_{l_{\beta}^{+}}\right)$for $\tilde{l}_{j}^{+} \rightarrow l_{\beta}^{+} \tilde{\chi}_{b}^{0}$ by the substitutions $B_{i \alpha, a} \rightarrow A_{j \beta, b}^{*}$ and $A_{i \alpha, a} \rightarrow B_{j \beta, b}^{*}[15]$.

\subsection{Cross-sections}

Having the amplitudes at hand it is straightforward to derive the cross-sections for the complete $2 \rightarrow 4$ processes $e^{ \pm} e^{-} \rightarrow \tilde{l}_{j}^{ \pm} \tilde{l}_{i}^{-} \rightarrow l_{\beta}^{ \pm} l_{\alpha}^{-} \tilde{\chi}_{b}^{0} \tilde{\chi}_{a}^{0}$. For the square of the amplitudes summed over all possible intermediate slepton states one finds

$$
\begin{aligned}
|M|^{2}= & \sum_{i j k l}\left(M_{i j} M_{k l}^{*}\right)\left(M_{i}^{-} M_{k}^{-*}\right)\left(M_{j}^{ \pm} M_{l}^{ \pm *}\right) C_{i k} \frac{\pi}{2 \overline{m \Gamma}_{i k}} C_{j l} \frac{\pi}{2 \overline{m \Gamma}_{j l}} \\
& \times\left(\delta\left(p_{3}^{2}-m_{\tilde{l}_{i}}^{2}\right)+\delta\left(p_{3}^{2}-m_{\tilde{l}_{k}}^{2}\right)\right)\left(\delta\left(p_{4}^{2}-m_{\tilde{l}_{j}}^{2}\right)+\delta\left(p_{4}^{2}-m_{\tilde{l}_{l}}^{2}\right)\right)
\end{aligned}
$$

with

$$
\begin{aligned}
C_{i k} & =\frac{1}{1+i \frac{\Delta \tilde{m}_{i k}^{2}}{2 m \Gamma_{i k}}}, \\
\overline{m \Gamma}_{i k} & =\frac{1}{2}\left(m_{\tilde{l}_{i}} \Gamma_{\tilde{l}_{i}}+m_{\tilde{l}_{k}} \Gamma_{\tilde{l}_{k}}\right), \quad \Delta \tilde{m}_{i k}^{2}=m_{\tilde{l}_{i}}^{2}-m_{\tilde{l}_{k}}^{2} .
\end{aligned}
$$


Here, we have used the narrow width approximation for the slepton propagators, which is well justified since the slepton widths $\Gamma_{\tilde{l}_{i}}$ are of order GeV or less, that is much smaller than the slepton masses of order $100 \mathrm{GeV}$. For the product of two slepton propagators, this approximation yields (see, e.g., [3])

$$
\left(\frac{1}{p^{2}-m_{\tilde{l}_{i}}^{2}+i m_{\tilde{l}_{i}} \Gamma_{\tilde{l}_{i}}}\right)\left(\frac{1}{p^{2}-m_{\tilde{l}_{k}}^{2}+i m_{\tilde{l}_{k}} \Gamma_{\tilde{l}_{k}}}\right)^{*} \approx C_{i k} \frac{\pi}{2 \overline{m \Gamma}_{i k}}\left(\delta\left(p^{2}-m_{\tilde{l}_{i}}^{2}\right)+\delta\left(p^{2}-m_{\tilde{l}_{k}}^{2}\right)\right) .
$$

Moreover, for such small slepton decay widths one can also neglect the interference between identical outgoing neutralinos, as we have checked numerically.

Integrating over the slepton momenta squared $p_{3}^{2}$ and $p_{4}^{2}$ and using the definitions

$$
\begin{aligned}
d \sigma_{i j k l} & =(2 \pi)^{4} \delta\left(p_{1}+p_{2}-p_{3}-p_{4}\right) \frac{d^{3} p_{3}}{(2 \pi)^{3} 2 E_{3}} \frac{d^{3} p_{4}}{(2 \pi)^{3} 2 E_{4}} \frac{M_{i j} M_{k l}^{*}}{2 s} \\
d B_{i k} & =(2 \pi)^{4} \delta\left(p_{3}-p_{5}-p_{7}\right) \frac{d^{3} p_{5}}{(2 \pi)^{3} 2 E_{5}} \frac{d^{3} p_{7}}{(2 \pi)^{3} 2 E_{7}} \frac{C_{i k}}{2 \overline{m \Gamma}_{i k}} M_{i}^{-} M_{k}^{-*} \\
d B_{j l} & =(2 \pi)^{4} \delta\left(p_{4}-p_{6}-p_{8}\right) \frac{d^{3} p_{6}}{(2 \pi)^{3} 2 E_{6}} \frac{d^{3} p_{8}}{(2 \pi)^{3} 2 E_{8}} \frac{C_{j l}}{2 \overline{2 \Gamma}_{j l}} M_{j}^{ \pm} M_{l}^{ \pm *}
\end{aligned}
$$

one can express the differential cross-sections in the form

$$
d \sigma=\frac{1}{4} \sum_{i j k l} \sum_{\substack{p_{3}^{2}=m_{\tilde{l}_{i}}^{2}, m_{\tilde{l}_{k}}^{2} \\ p_{4}^{2}=m_{\tilde{l}_{j}}^{2}, m_{\tilde{l}_{l}}^{2}}} d \sigma_{i j k l} d B_{i k} d B_{j l} .
$$

As one expects on general grounds, for large mass differences, $\Delta \tilde{m}_{i k}^{2} \gg \overline{m \Gamma}_{i k}$, the factors $C_{i k}$ in (33) approach $\delta_{i k}$. Consequently, the coherent sum in (37) reduces to an incoherent sum over products of production cross-sections times branching ratios:

$$
d \sigma=\sum_{i j} d \sigma\left(e^{ \pm} e^{-} \rightarrow \tilde{l}_{j}^{ \pm} \tilde{l}_{i}^{-}\right) d B r\left(\tilde{l}_{i}^{-} \rightarrow l_{\alpha}^{-} \tilde{\chi}_{a}^{0}\right) d B r\left(\tilde{l}_{j}^{ \pm} \rightarrow l_{\beta}^{ \pm} \tilde{\chi}_{b}^{0}\right) .
$$

Previous studies often used only generic slepton masses and widths. In the present analysis the slepton decay widths $\Gamma_{\tilde{l}_{i}}$ are actually calculated for each of the mSUGRA benchmark scenarios, including the effects of LFV. This is important for consistent phenomenological studies.

\section{Numerical results}

\subsection{SUSY and neutrino parameters}

Similarly as shown in [7] for the radiative decays $l_{\alpha} \rightarrow l_{\beta} \gamma, \mathrm{LFV}$ in $e^{ \pm} e^{-} \rightarrow l_{\beta}^{ \pm} l_{\alpha}^{-} \tilde{\chi}_{b}^{0} \tilde{\chi}_{a}^{0}$ depends very sensitively on the SUSY scenario and on the neutrino masses and mixings. 
The choice of models and the neutrino data are already described in some detail in the article mentioned above. Therefore, it suffices here to briefly specify the input parameters used in the following numerical analysis.

Again, we choose the mSUGRA benchmark scenarios proposed in [8] for linear collider studies. In these set of models the universal trilinear coupling parameter $A_{0}$ is assumed to vanish and, hence, LVF only occurs in the left-handed slepton sector as can be seen from (12)-(14). For this reason, we focus on those scenarios which lead to sufficiently light sleptons, so that the production of slepton pairs containing a large admixture of at least one left-handed $\tilde{l}_{L}$ component is possible at $\sqrt{s}=500 \mathrm{GeV}$. The corresponding values of the mSUGRA parameters are listed in Tab. 1 together with some characteristic predictions.

\begin{tabular}{|c|c|c|c||c|c|c|}
\hline Scenario & $m_{1 / 2} / \mathrm{GeV}$ & $m_{0} / \mathrm{GeV}$ & $\tan \beta$ & $m_{\tilde{l}_{6}} / \mathrm{GeV}$ & $\Gamma_{\tilde{l}_{6}} / \mathrm{GeV}$ & $m_{\tilde{\chi}_{1}^{0}} / \mathrm{GeV}$ \\
\hline \hline $\mathrm{B}$ & 250 & 100 & 10 & 208 & 0.32 & 98 \\
\hline $\mathrm{C}$ & 400 & 90 & 10 & 292 & 0.22 & 164 \\
\hline $\mathrm{G}$ & 375 & 120 & 20 & 292 & 0.41 & 154 \\
\hline $\mathrm{I}$ & 350 & 180 & 35 & 313 & 1.03 & 143 \\
\hline
\end{tabular}

Table 1: Parameters of the relevant mSUGRA benchmark scenarios (from [8]). The sign of $\mu$ is chosen to be positive and $A_{0}$ is set to zero. Given are also the mass and total width of the heaviest charged slepton and the mass of the lightest neutralino.

Most likely, at the time when a linear collider will be in operation, more precise measurements of the neutrino parameters will be available than today. In order to simulate the expected improvement, we take the central values of the mass squared differences $\Delta m_{i j}^{2}=m_{j}^{2}-m_{i}^{2}$ and mixing angles $\theta_{i j}$ from a global fit to existing data [18, with errors that indicate the anticipated $90 \%$ C.L. intervals of running and proposed experiments as further explained in [7:

$$
\begin{aligned}
& \tan ^{2} \theta_{23}=1.40_{-0.66}^{+1.37}, \quad \tan ^{2} \theta_{13}=0.005_{-0.005}^{+0.001}, \quad \tan ^{2} \theta_{12}=0.36_{-0.16}^{+0.35}, \\
& \Delta m_{12}^{2}=3.30_{-0.3}^{+0.3} \cdot 10^{-5} \mathrm{eV}^{2}, \quad \Delta m_{23}^{2}=3.10_{-1.0}^{+1.0} \cdot 10^{-3} \mathrm{eV}^{2} .
\end{aligned}
$$

The fit [18] is not the latest one, but still valid within the present uncertainties. It is taken for consistency with the results derived in [7] and used in the following. ${ }^{2}$ The CP-violating

\footnotetext{
${ }^{2}$ In section 4.2 we will remark on the slight changes in our results when using the central values of the neutrino parameters from one of the more recent fits 19 instead of 18 .
} 
Dirac phase $\delta$ is very difficult to measure and hence we allow it to vary in the full range

$$
\delta=0-2 \pi
$$

Furthermore, we consider both hierarchical and quasi-degenerate spectra of light neutrinos. The case of neutrino masses that are too light for an absolute mass measurement, is described by

$$
m_{1}=0-0.03 \mathrm{eV}, \quad m_{2}=\sqrt{m_{1}^{2}+\Delta m_{12}^{2}}, \quad m_{3}=\sqrt{m_{2}^{2}+\Delta m_{23}^{2}} .
$$

For $m_{1} \ll m_{2} \ll m_{3}$, the combination $Y_{\nu}^{\dagger} Y_{\nu}$, entering via (17) the renormalization group correction (12) to the slepton mass matrix, can be approximated by

$$
\left(Y_{\nu}^{\dagger} Y_{\nu}\right)_{i j} \approx \frac{M_{R}}{v^{2} \sin ^{2} \beta}\left(\sqrt{\Delta m_{12}^{2}} U_{i 2} U_{j 2}^{*}+\sqrt{\Delta m_{23}^{2}} U_{i 3} U_{j 3}^{*}\right) .
$$

For the quasi-degenerate case we take

$$
m_{1}=\left(0.3_{-0.16}^{+0.11}\right) \mathrm{eV}, \quad m_{2} \approx m_{1}+\frac{1}{2 m_{1}} \Delta m_{12}^{2}, \quad m_{3} \approx m_{1}+\frac{1}{2 m_{1}} \Delta m_{23}^{2} .
$$

Since in this case $m_{1} \gg \sqrt{\Delta m_{12}^{2}}, \sqrt{\Delta m_{23}^{2}}$, one can use the approximation

$$
\left(Y_{\nu}^{\dagger} Y_{\nu}\right)_{i j} \approx \frac{M_{R}}{v^{2} \sin ^{2} \beta}\left(m_{1} \delta_{i j}+\left(\frac{\Delta m_{12}^{2}}{2 m_{1}} U_{i 2} U_{j 2}^{*}+\frac{\Delta m_{23}^{2}}{2 m_{1}} U_{i 3} U_{j 3}^{*}\right)\right) .
$$

The Majorana mass $M_{R}$ is kept as a free parameter and is only constrained by the requirement that $Y_{\nu}$ should stay small enough for perturbation theory to hold.

\subsection{Predictions for $e^{+} e^{-}$and $e^{-} e^{-}$collisions}

Using the numerical input specified in the preceding subsection, we have calculated the cross-sections for $e^{+} e^{-} \rightarrow \tilde{l}_{j}^{+} \tilde{l}_{i}^{-} \rightarrow \mu^{+} e^{-}+2 \tilde{\chi}_{1}^{0}$ and $\tau^{+} \mu^{-}+2 \tilde{\chi}_{1}^{0}$, as well as the cross-section for $e^{-} e^{-} \rightarrow \tilde{l}_{j}^{-} \tilde{l}_{i}^{-} \rightarrow \mu^{-} e^{-}+2 \tilde{\chi}_{1}^{0}$. In the SUSY scenarios under study R-parity is preserved, and $\tilde{\chi}_{1}^{0}$ is the lightest SUSY particle and therefore stable. Since $\tilde{\chi}_{1}^{0}$ is not detected, the observable final states consist of a pair of charged leptons with different flavor plus missing energy $(\not E)$. Thus one is dealing with a rather simple signal. Note that in the models defined in Tab. [ the heavier neutralino states $\tilde{\chi}_{a}^{0}, a=2,3,4$ do not contribute to these signals: either they are too heavy and hence not or only very rarely produced in the slepton decays, or they are too light in order to decay invisibly via $\tilde{\chi}_{a}^{0} \rightarrow \tilde{\nu} \nu \rightarrow \tilde{\chi}_{1}^{0} \nu \nu$. Other open channels such as $\tilde{\tau} \tau$ lead to more complicated final states which are not considered here. As a rule of 
thumb, for an integrated luminosity of $1000 \mathrm{fb}^{-1}$, the goal at an $e^{+} e^{-}$LC such as TESLA, the cross-section should be larger than $10^{-2} \mathrm{fb}$ for a signal to become observable.

Fig. 3] shows the cross-sections for $\mu^{+} e^{-}+2 \tilde{\chi}_{1}^{0}$ and $\tau^{+} \mu^{-}+2 \tilde{\chi}_{1}^{0}$ at $\sqrt{s}=500 \mathrm{GeV}$ as a function of the right-handed Majorana mass scale $M_{R}$ in scenario B which predicts the lightest sleptons. We have assumed a very light neutrino spectrum and have scattered the neutrino parameters in the error intervals specified in (39)-(42) according to Gaussian distributions, except $m_{1}$ and $\delta$ for which we have taken flat distributions. Each choice corresponds to one particular point in Fig. 3.

A few comments concerning the main features of this plot are in order. Similarly as the branching ratios $\operatorname{Br}\left(l_{\alpha} \rightarrow l_{\beta} \gamma\right)$ [7], the cross-sections exhibit the typical proportionality to $\left(Y_{\nu}^{\dagger} Y_{\nu}\right)^{2} \propto M_{R}^{2}$. This behavior indicates that the signal cross-sections are dominated by Feynman graphs involving the lepton-flavor violating vertices in first order. The latter in turn implies that $e^{+} e^{-} \rightarrow \tau^{+} \mu^{-}+2 \tilde{\chi}_{1}^{0}$ is dominantly a $s$-channel process and thus suppressed relative to $e^{+} e^{-} \rightarrow \mu^{+} e^{-}+2 \tilde{\chi}_{1}^{0}$ where the $s$ - and $t$-channel contribute.

Furthermore, in contrast to the branching ratios of the radiative decays, the cross-sections saturate for large $M_{R}$. This saturation is seen most clearly in the $\mu^{+} e^{-}$channel. It can be understood by realizing that for large $M_{R}$ the mass differences of the sleptons with a dominant left-handed component become comparable to the corresponding slepton widths. As already mentioned, in this case the cross-sections can be approximated by the incoherent sum (38). Focussing on the $e \mu$ channel, the dominant contribution is determined by the $\tilde{e}_{L}-\tilde{\mu}_{L}$ mixing angle or, more precisely, by

$$
\tan 2 \tilde{\theta}_{e \mu} \approx \frac{2\left(m_{\tilde{l}_{L}}^{2}\right)_{12}}{\left(m_{\tilde{l}_{L}}^{2}\right)_{11}-\left(m_{\tilde{l}_{L}}^{2}\right)_{22}} \approx \frac{2\left(Y_{\nu}^{\dagger} Y_{\nu}\right)_{12}}{\left(Y_{\nu}^{\dagger} Y_{\nu}\right)_{11}-\left(Y_{\nu}^{\dagger} Y_{\nu}\right)_{22}},
$$

where the second equality follows from (6), (9), and (12) if the lepton masses are neglected. Using (43) one then sees that $\tan 2 \tilde{\theta}_{e \mu}$ and thus also the cross-sections are independent of $M_{R}$. In the $\tau^{+} \mu^{-}$channel the saturation is less pronounced due to effects of the heavier $\tau$ mass which dominate the denominator in the expression analogous to (46), except for very large values of $M_{R}$. The finite $\tau$ mass is also responsible for a suppression of all channels with a $\tau$ lepton in the final state relative to the $\mu e$ channel.

Finally, the impact of the neutrino uncertainties is weaker in the $\tau^{+} \mu^{-}$than in the $\mu^{+} e^{-}$ channel. This is not surprising, since the product of neutrino Yukawa couplings $\left(Y_{\nu}^{\dagger} Y_{\nu}\right)_{23}$ relevant for $\tau^{+} \mu^{-}$is mainly given by a single term depending on the large angle $\theta_{23}$ and mass squared difference $\Delta m_{23}^{2}$, whereas $\left(Y_{\nu}^{\dagger} Y_{\nu}\right)_{12}$ relevant for $\mu^{+} e^{-}$depends on two terms involving the small quantities $\theta_{13}$ and $\Delta m_{12}^{2}$, respectively, as can be seen in (43) and (45) 
for $i \neq j$. Given the uncertainties in $\theta_{13}$, the two terms can become similar in magnitude and cancel depending on the unknown phase $\delta$. The same holds for the $\tau e$ channel. Since this channel is unfavorable because of the $\tau$ mass suppression pointed out above and the comparably large MSSM background, we will concentrate on the $\mu e$ and $\tau \mu$ final states. The corresponding cross-sections for the higher cms energy $\sqrt{s}=800 \mathrm{GeV}$ are plotted in Fig. 4. Here, the scenario I being unaccessible at $500 \mathrm{GeV}$ is included. This figure illustrates the range of cross-sections which can be expected in the benchmark models specified in Tab. 1. Together with Fig. 3 it also indicates the energy-dependence of the signals.

If the central values of the neutrino parameters from one of the latest fits [19] are used instead of (39) and (40), the most likely predictions for $\sigma\left(e^{+} e^{-} \rightarrow \mu^{+} e^{-}+2 \tilde{\chi}_{1}^{0}\right)$ rise by about $30 \%$ as compared to the results shown on Fig. 3 and 4 , while the spread of the predictions shrinks slightly. Furthermore, the cross sections for $e^{+} e^{-} \rightarrow \tau^{+} \mu^{-}+2 \tilde{\chi}_{1}^{0}$ decrease by about $20 \%$ with the spread remaining unchanged.

We now turn to a particularly interesting subject, namely the correlations between the cross-sections $\sigma\left(e^{ \pm} e^{-} \rightarrow \tilde{l}_{j}^{ \pm} \tilde{l}_{i}^{-} \rightarrow l_{\beta}^{ \pm} l_{\alpha}^{-} \tilde{\chi}_{b}^{0} \tilde{\chi}_{a}^{0}\right)$ and the branching ratios $\operatorname{Br}\left(l_{\alpha} \rightarrow l_{\beta} \gamma\right)$. This correlation is illustrated in Fig. 5 for $e^{+} e^{-} \rightarrow \mu^{+} e^{-}+2 \tilde{\chi}_{1}^{0}$ at $\sqrt{s}=500 \mathrm{GeV}$ and $\mu \rightarrow e \gamma$. For $M_{R} \lesssim 10^{13} \mathrm{GeV}$, corresponding roughly to $\operatorname{Br}(\mu \rightarrow e \gamma) \lesssim 10^{-13}$ [7], this correlation is so accurate that the neutrino uncertainties drop out almost completely. Only for larger values of $M_{R}$ the correlation gets lost because of the saturation of the cross-sections explained above which sets in at different values of $M_{R}$ depending on the precise values of the neutrino parameters. Fig. [6] shows the analogous relation between $\sigma\left(e^{+} e^{-} \rightarrow \tau^{+} \mu^{-}+2 \tilde{\chi}_{1}^{0}\right)$ and $\operatorname{Br}(\tau \rightarrow \mu \gamma)$ which is even stronger. Note that beyond the upper ends of the scatter plots in Fig. [6] the neutrino Yukawa couplings would become too large for perturbation theory to hold.

These correlations can be used to estimate the LC cross-sections that are allowed by bounds on or measurements of the radiative decays. Taking scenario B as an example, one can read off from Fig. [5] that the present bound $\operatorname{Br}(\mu \rightarrow e \gamma)<1.2 \cdot 10^{-11}\left[20\right.$ implies $\sigma\left(e^{+} e^{-} \rightarrow\right.$ $\left.\mu^{+} e^{-}+2 \tilde{\chi}_{1}^{0}\right)<0.3 \mathrm{fb}$, while a measurement of $\operatorname{Br}(\mu \rightarrow e \gamma) \approx 10^{-14}$ by the new experiment at PSI [21] would predict $\sigma\left(e^{+} e^{-} \rightarrow \mu^{+} e^{-}+2 \tilde{\chi}_{1}^{0}\right) \approx 3 \cdot 10^{-4} \mathrm{fb}$. In other words, if $\mu \rightarrow e \gamma$ will not be detected at PSI, one does not expect an observable $\mu e$ signal at a $500 \mathrm{GeV} \mathrm{LC}$ either. In model $\mathrm{C}$, the above branching ratios imply $\sigma\left(e^{+} e^{-} \rightarrow \mu^{+} e^{-}+2 \tilde{\chi}_{1}^{0}\right)<1 \mathrm{fb}$ and $\approx 6 \cdot 10^{-3} \mathrm{fb}$, respectively.

Analogously, from Fig. [6] one concludes that the present bound $\operatorname{Br}(\tau \rightarrow \mu \gamma)<6 \cdot 10^{-7}$ 22. provides the weak constraints $\sigma\left(e^{+} e^{-} \rightarrow \tau^{+} \mu^{-}+2 \tilde{\chi}_{1}^{0}\right)<10 \mathrm{fb}$ at $\sqrt{s}=800 \mathrm{GeV}$ for 
model B, C, and G, and $<2$ fb for model I. More importantly, also the sensitivity goal $\operatorname{Br}(\tau \rightarrow \mu \gamma) \approx 10^{-9}$ of future searches [23] will not rule out sizable LC cross-sections, namely $\sigma\left(e^{+} e^{-} \rightarrow \tau^{+} \mu^{-}+2 \tilde{\chi}_{1}^{0}\right) \approx 6(0.2) \mathrm{fb}$ in scenario $\mathrm{C}$ (B and $\mathrm{G}$ ). In fact, in the $\tau \mu$ channel LC experiments may reach farther than the $\tau \rightarrow \mu \gamma$ experiments planned in the future. At any rate, here we have a nice example for the complementarity of low and high-energy searches.

In principle, one has such correlations also in channels which differ in flavor. This is exemplified in Fig. [7 for $\sigma\left(e^{+} e^{-} \rightarrow \tau^{+} \mu^{-}+2 \tilde{\chi}_{1}^{0}\right)$ and $\operatorname{Br}(\mu \rightarrow e \gamma)$. However, because of the different flavor-violating couplings involved this correlation suffers considerably from uncertainties in the neutrino sector. Nevertheless, the experimental bound $\operatorname{Br}(\mu \rightarrow e \gamma)<1.2 \cdot 10^{-11}$ yields a stronger constraint on model I than the one obtained from Fig. 6, excluding $\sigma\left(\tau^{+} \mu^{-}+2 \tilde{\chi}_{1}^{0}\right)>$ few $\cdot 10^{-2}$ fb at $\sqrt{s}=800 \mathrm{GeV}$ already today.

Finally, it is interesting to look at the relation between the two cross-sections $\sigma\left(e^{+} e^{-} \rightarrow\right.$ $\left.\tau^{+} \mu^{-}+2 \tilde{\chi}_{1}^{0}\right)$ and $\sigma\left(e^{+} e^{-} \rightarrow \mu^{+} e^{-}+2 \tilde{\chi}_{1}^{0}\right)$ themselves. This relation is displayed in Fig. 8 for $800 \mathrm{GeV}$ and scenario C. Unfortunately, it is washed out by the uncertainties in the neutrino parameters. Nevertheless, a measurement in the $\mu e$ channel would give a useful lower bound in the $\tau \mu$ channel.

The use of the latest central values of the neutrino parameters from [19] instead of (39) and (40) does not change the correlations shown in Fig. 5 and 6 significantly. On the other hand, the lower boundaries of the correlations displayed in Fig. 7 and 8 are slightly shifted to the right.

For completeness, we have also studied the prospects for $e^{-} e^{-}$collisions. Fig. 9 shows $\sigma\left(e^{-} e^{-} \rightarrow \mu^{-} e^{-}+2 \tilde{\chi}_{1}^{0}\right)$ and $\sigma\left(e^{-} e^{-} \rightarrow \tau^{-} \mu^{-}+2 \tilde{\chi}_{1}^{0}\right)$ for $\sqrt{s}=800 \mathrm{GeV}$ and model C. The strong suppression of the $\tau^{-} \mu^{-}$final state results from the fact that at least two flavor-violating couplings are required because of the absence of the $s$-channel (see Fig. 2). The $\tau^{-} e^{-}$final state is suppressed for similar reasons as the $\tau^{+} e^{-}$channel in $e^{+} e^{-}$ collisions. Thus, $\mu^{-} e^{-}$remains as the only promising channel. The correlation between $\sigma\left(e^{-} e^{-} \rightarrow \mu^{-} e^{-}+2 \tilde{\chi}_{1}^{0}\right)$ and $\operatorname{Br}(\mu \rightarrow e \gamma)$ is plotted in Fig. 10 for the same scenario C. We see that the cross-section permitted by the present bound on $\mu \rightarrow e \gamma$ lies in the range of 1-10 fb which would make the detection rather easy. In this figure, we also show the prediction for a degenerate neutrino spectrum. Generally, both the LC cross-sections as well as the radiative branching ratios tend to be smaller for degenerate than for hierarchical neutrinos. This reduction comes from the suppression of the lepton-flavor violating terms in (45) by the neutrino mass $m_{1}$ which sets the absolute mass scale. However, the correlation 
between cross-sections and branching ratios is found to be more or less independent of the absolute neutrino mass scale. The predictions shown in Fig. 9 would change only slightly, if the central values of the neutrino parameters from [19] are used instead of (39) and (40), similarly as described above for $e^{+} e^{-}$scattering. Again, the correlation depicted in Fig. 10 remains unaffected.

\section{$5 \quad$ Background processes}

After having discussed the lepton-flavor violating signals leading to the channels $l_{\beta} l_{\alpha}+\mathbb{E}$ we turn now to the lepton-flavor conserving standard model (SM) and SUSY background. For the dominant SM reactions we also illustrate the efficiency of angular and energy cuts in reducing this background. The SUSY background processes are calculated in the respective mSUGRA benchmark scenarios without cuts. We are going to assume that at the time these searches for LFV will be performed, SUSY has already been discovered and the lighter sparticle masses and main decay channels are known. In this case, one will be able to design very specific cuts optimized to each channel of interest. Under this assumption our estimates indicate that it should be possible to reduce the SM and SUSY background sufficiently and detect some of the signals considered, provided the Majorana scale $M_{R}$ is not much smaller than $10^{13} \mathrm{GeV}$. However, more conclusive statements on the feasibility to discriminate signal from background require detailed simulations which are beyond the scope of this paper.

\subsection{Background in $e^{+} e^{-}$}

In the SM, the dominant background in the channels $e^{+} e^{-} \rightarrow \tilde{l}_{j}^{+} \tilde{l}_{i}^{-} \rightarrow l_{\beta}^{+} l_{\alpha}^{-}+2 \tilde{\chi}_{1}^{0}$ is produced by the following lepton-flavor conserving processes:

B1) $e^{+} e^{-} \rightarrow W^{+} W^{-} \rightarrow l_{\beta}^{+} \nu_{\beta} l_{\alpha}^{-} \bar{\nu}_{\alpha} \quad(\alpha \neq \beta)$,

B2) $e^{+} e^{-} \rightarrow W^{+} e^{-} \bar{\nu}_{e} \rightarrow l_{\beta}^{+} \nu_{\beta} e^{-} \bar{\nu}_{e} \quad(\beta \neq 1)$,

B3) $e^{+} e^{-} \rightarrow \tau^{+} \tau^{-} \rightarrow \tau^{+} \nu_{\tau} l_{\alpha}^{-} \bar{\nu}_{\alpha} \quad(\alpha=1,2)$.

These have been estimated with the help of the COMPHEP program package [24], including the following kinematical cuts:

i) beam-pipe cut: $\left|\cos \left(e^{ \pm}, l_{\beta, \alpha}^{ \pm}\right)\right|<0.985\left(10^{\circ}\right)$, 
ii) lepton-energy cut: $E_{\min } \leq E_{l} \leq E_{\max }$,

iii) missing-energy cut: $2 m_{\tilde{\chi}_{1}^{0}} \leq \mathscr{E} \leq \sqrt{s}-2 E_{\min }$.

The angular cut i) partially eliminates the large contribution from $t$-channel photon exchange (B2), and also small-angle $W$ (B1) and $\tau$ (B3) production. Since the angular distributions of the decay leptons from the heavy sleptons are relatively flat, the signal rates are thereby reduced by less than $10 \%$. Then, knowing the slepton spectrum and the LSP mass, one can impose the cuts (ii) and (iii) requiring the lepton energies and the total missing energy to lie in the intervals corresponding to the decay $\tilde{l} \rightarrow l \tilde{\chi}_{1}^{0}$. Since one is dealing with a coherent process, the intermediate slepton flavor is not known. Therefore, $E_{\min }\left(E_{\max }\right)$ is defined as the minimum (maximum) of the decay energies kinematically allowed for any slepton flavor:

$$
E_{\min }=\min \left(\frac{m_{\tilde{l}}^{2}-m_{\tilde{\chi}_{1}^{0}}^{2}}{2\left(E_{\tilde{l}}+p_{\tilde{l}}\right)}\right), E_{\max }=\max \left(\frac{m_{\tilde{l}}^{2}-m_{\tilde{\chi}_{1}^{0}}^{2}}{2\left(E_{\tilde{l}}-p_{\tilde{l}}\right)}\right)
$$

\begin{tabular}{|c|c|c|c||c|c|c|}
\hline Final state & $\sqrt{s} / \mathrm{GeV}$ & Cut & $\sigma / f b$ & $\sqrt{s} / \mathrm{GeV}$ & Cut & $\sigma / f b$ \\
\hline$\mu^{+} e^{-} \bar{\nu}_{e} \nu_{\mu}$ & 500 & $\mathrm{i}$ & 105.5 & 800 & $\mathrm{i}$ & 85.1 \\
\hline & & $\mathrm{i}+\mathrm{ii}+\mathrm{iii}, \mathrm{B}$ & 29.6 & & $\mathrm{i}+\mathrm{ii}+\mathrm{iii}, \mathrm{B}$ & 27.5 \\
\hline & & $\mathrm{i}+\mathrm{ii}+\mathrm{iii}, \mathrm{C}$ & 9.2 & & $\mathrm{i}+\mathrm{ii}+\mathrm{iii}, \mathrm{C}$ & 19.0 \\
\hline & & $\mathrm{i}+\mathrm{ii}+\mathrm{iii}, \mathrm{G}$ & 11.6 & & $\mathrm{i}+\mathrm{ii}+\mathrm{iii}, \mathrm{G}$ & 20.8 \\
\hline & & $\mathrm{i}+\mathrm{ii}+\mathrm{iii}, \mathrm{I}$ & 10.8 & & $\mathrm{i}+\mathrm{ii}+\mathrm{iii}, \mathrm{I}$ & 24.2 \\
\hline \hline Final state & $\sqrt{s} / \mathrm{GeV}$ & $\mathrm{Cut}$ & $\sigma / f b$ & $\sqrt{s} / \mathrm{GeV}$ & $\mathrm{Cut}$ & $\sigma / f b$ \\
\hline$\tau^{+} \mu^{-} \bar{\nu}_{\mu} \nu_{\tau}$ & 500 & $\mathrm{i}$ & 58.8 & 800 & $\mathrm{i}$ & 22.5 \\
\hline & & $\mathrm{i}+\mathrm{ii}+\mathrm{iii}, \mathrm{B}$ & 11.1 & & $\mathrm{i}+\mathrm{ii}+\mathrm{iii}, \mathrm{B}$ & 6.0 \\
\hline & & $\mathrm{i}+\mathrm{ii}+\mathrm{iii}, \mathrm{C}$ & 3.1 & & $\mathrm{i}+\mathrm{ii}+\mathrm{iii}, \mathrm{C}$ & 3.5 \\
\hline & & $\mathrm{i}+\mathrm{ii}+\mathrm{iii}, \mathrm{G}$ & 3.5 & & $\mathrm{i}+\mathrm{ii}+\mathrm{iii}, \mathrm{G}$ & 3.9 \\
\hline & & $\mathrm{i}+\mathrm{ii}+\mathrm{iii}, \mathrm{I}$ & 3.6 & & $\mathrm{i}+\mathrm{ii}+\mathrm{iii}, \mathrm{I}$ & 4.8 \\
\hline
\end{tabular}

Table 2: Cross-sections for the SM background in $e^{+} e^{-}$collisions including cuts as explained in the text.

The SM background cross-sections that remain after these cuts are summarized in Tab. 2] If one requires a signal to background ratio $S / \sqrt{B}=2$ and assumes a typical signal crosssection of $0.1 \mathrm{fb}$, one can afford a background of about $2 \mathrm{fb}$. The additional suppression may 
be achieved by applying the standard selectron selection cuts [25] on the acoplanarity, lepton polar angle and missing transverse momentum. It has been shown that in this way the SM background to slepton-pair production can be reduced to about $2-3 \mathrm{fb}$ at $\sqrt{s}=500 \mathrm{GeV}$, while the signal cross-section shrinks only by a factor 3 . It should be noted that suppression of the $W$-pair background by right-handed electron polarization is not an option here, since in the mSUGRA scenarios considered LFV occurs only in the left-handed slepton sector, and thus right-handed polarization would also kill the signal.

In the MSSM, the main background processes are

B4) $e^{+} e^{-} \rightarrow \tilde{\nu}_{\alpha} \overline{\tilde{\nu}}_{\alpha}$

B5) $e^{+} e^{-} \rightarrow \tilde{l}_{\alpha}^{+} \tilde{l}_{\alpha}^{-}$,

B6) $e^{+} e^{-} \rightarrow \tilde{\chi}_{b}^{+} \tilde{\chi}_{a}^{-}$,

B7) $e^{+} e^{-} \rightarrow \tilde{\chi}_{b}^{+} e^{-} \overline{\tilde{\nu}}_{e}$,

with the sparticles decaying like $\tilde{\nu}_{\alpha} \rightarrow \nu_{\alpha} \tilde{\chi}_{1}^{0}$ and $l_{\alpha}^{-} \tilde{\chi}_{1}^{+}, \tilde{\chi}_{1}^{+} \rightarrow l_{\alpha}^{+} \tilde{\nu}_{\alpha}$ and $\tilde{l}_{\alpha}^{+} \nu_{\alpha}, \tilde{l}_{\alpha}^{+} \rightarrow l_{\alpha}^{+} \tilde{\chi}_{1}^{0}$ and $\bar{\nu}_{\alpha} \tilde{\chi}_{1}^{+}$, and many other channels, depending on the given sparticle spectrum.

The numerical estimates for the MSSM background listed in Tab. 3] are again obtained with the help of COMPHEP. Here, no cuts are included and the cross-sections for the individual production channels are added incoherently. We find that the MSSM background to $\mu^{+} e^{-}+\not E$ is very small, below $0.2 \mathrm{fb}$ in all scenarios of Tab. 1 except for model I and $\mathrm{C}$, where it amounts to 0.4 and $5 \mathrm{fb}$, respectively, at $\sqrt{s}=800 \mathrm{GeV}$. With $2-7 \mathrm{fb}$ the $\tau^{+} \mu^{-}$background is considerably bigger. Also shown in Tab. 3 is the MSSM background to $\tau^{+} e^{-}+\not E$, which can contribute to the $\mu^{+} e^{-}$channel via the decay $\tau^{+} \rightarrow \mu^{+} \nu_{\mu} \bar{\nu}_{\tau}$. If $\tilde{\tau}_{1}$ and $\tilde{\chi}_{1}^{+}$are very light, like in scenarios B and I, this background can be as large as $100 \mathrm{fb}$. The charginos originate mainly from selectron or $\tilde{\nu}_{e}$ pair production and decay almost exclusively into staus, $\tilde{\chi}_{1}^{-} \rightarrow \tilde{\tau}_{1}^{-} \bar{\nu}_{\tau}$. However, such events typically contain two neutrinos in addition to the two LSPs which are also present in the signal events. Thus, after $\tau$ decay one has altogether six invisible particles instead of two, which should allow to discriminate the signal in $\mu^{+} e^{-}+\not E$ also from this potentially dangerous MSSM background by cutting on various distributions.

\subsection{Background in $e^{-} e^{-}$}

In seesaw models the process $e^{-} e^{-} \rightarrow W^{-} W^{-} \rightarrow l_{\beta}^{-} l_{\alpha}^{-} \bar{\nu}_{\beta} \bar{\nu}_{\alpha}(\alpha, \beta \neq 1)$ via neutrino exchange is highly suppressed due to the very small admixture of the heavy Majorana neutrinos in 


\begin{tabular}{|c|c|c|c|c|c|c|c|}
\hline Final state & Production & \multicolumn{2}{|c|}{$\mathrm{B}$} & $\mathrm{C}$ & \multicolumn{2}{|c|}{$\mathrm{G}$} & $\mathrm{I}$ \\
\hline$\mu^{+} e^{-}+\not{E}$ & & 500 & 800 & 800 & 500 & 800 & 800 \\
\hline & $\tilde{l}_{i}^{+} \tilde{l}_{i}^{-}$ & 0 & 0 & 0 & 0.02 & 0.06 & 0.1 \\
\hline & $\tilde{\tilde{\nu}}_{i} \tilde{\nu}_{i}$ & 0.01 & 0.01 & 0 & - & 0 & 0.3 \\
\hline & $\tilde{\chi}_{b}^{+} \tilde{\chi}_{a}^{-}$ & 0 & 0.07 & 2.5 & - & 0.05 & 0.01 \\
\hline & $\tilde{\chi}_{1}^{+} e^{-} \bar{\nu}_{e}$ & 0 & 0.04 & 2.3 & - & 0.07 & 0 \\
\hline & Sum & 0.01 & 0.12 & 4.8 & 0.02 & 0.18 & 0.41 \\
\hline \hline$\tau^{+} \mu^{-}+\not E$ & & 500 & 800 & 800 & 500 & 800 & 800 \\
\hline & $\tilde{l}_{i}^{+} \tilde{l}_{i}^{-}$ & 3.5 & 4.4 & 0 & 0 & 0.38 & 2.0 \\
\hline & $\tilde{\nu}_{i} \tilde{\nu}_{i}$ & 1.8 & 1.6 & 0.09 & - & 0.19 & 1.2 \\
\hline & $\tilde{\chi}_{b}^{+} \tilde{\chi}_{a}^{-}$ & 0.02 & 1.3 & 5.9 & - & 1.7 & 0.8 \\
\hline & Sum & 5.3 & 7.3 & 6.0 & 0 & 2.3 & 4.0 \\
\hline \hline$\tau^{+} e^{-}+\not E$ & & 500 & 800 & 800 & 500 & 800 & 800 \\
\hline & $\tilde{l}_{i}^{+} \tilde{l}_{i}^{-}$ & 38.2 & 37.5 & 0 & 0.8 & 2.0 & 16.2 \\
\hline & $\tilde{\nu}_{i} \tilde{\nu}_{i}$ & 60.0 & 81.5 & 0.09 & - & 0.2 & 38.7 \\
\hline & $\tilde{\chi}_{b}^{+} \tilde{\chi}_{a}^{-}$ & 0 & 0.8 & 5.9 & - & 1.7 & 0.5 \\
\hline & $\tilde{\chi}_{1}^{+} e^{-} \tilde{\nu}_{e}$ & 0 & 0.04 & 1.7 & - & 0.05 & 0 \\
\hline & Sum & 98.2 & 119.8 & 7.7 & 0.8 & 4.0 & 55.4 \\
\hline
\end{tabular}

Table 3: Cross-sections in fb for the MSSM background in $e^{+} e^{-}$collisions at $\sqrt{s}=500 \mathrm{GeV}$ and $800 \mathrm{GeV}$. The second column specifies the important production channels. In scenarios $\mathrm{C}$ and I the cross-sections are below $0.01 \mathrm{fb}$ at $\sqrt{s}=500 \mathrm{GeV}$ and are therefore omitted. Otherwise, cross-sections below $0.01 \mathrm{fb}$ are denoted by 0 . Kinematically forbidden channels are marked by a hyphen. 


\begin{tabular}{|c|c|c||c|c|c|c|}
\hline Final state & $\sqrt{s} / \mathrm{GeV}$ & Cut & $\sigma / f b$ & $\sqrt{s} / \mathrm{GeV}$ & Cut & $\sigma / f b$ \\
\hline$e^{-} \mu^{-} \nu_{e} \bar{\nu}_{\mu}$ & 500 & $\mathrm{i}$ & 122.8 & 800 & $\mathrm{i}$ & 175.7 \\
\hline & & $\mathrm{i}+\mathrm{ii}+\mathrm{iii}, \mathrm{B}$ & 55.5 & & $\mathrm{i}+\mathrm{ii}+\mathrm{iii}, \mathrm{B}$ & 79.5 \\
\hline & & $\mathrm{i}+\mathrm{ii}+\mathrm{iii}, \mathrm{C}$ & 13.9 & & $\mathrm{i}+\mathrm{ii}+\mathrm{iii}, \mathrm{C}$ & 55.2 \\
\hline & & $\mathrm{i}+\mathrm{ii}+\mathrm{iii}, \mathrm{G}$ & 17.8 & & $\mathrm{i}+\mathrm{ii}+\mathrm{iii}, \mathrm{G}$ & 61.1 \\
\hline & & $\mathrm{i}+\mathrm{ii}+\mathrm{iii}, \mathrm{I}$ & 19.4 & & $\mathrm{i}+\mathrm{ii}+\mathrm{iii}, \mathrm{I}$ & 70.8 \\
\hline
\end{tabular}

Table 4: Cross-sections for SM background processes in $e^{-} e^{-}$collisions including cuts as explained in the text.

the light neutrino eigenstates [26]. The analogous argument holds for the SUSY version of this process, i.e. chargino-pair production via $t$-channel sneutrino exchange. The main background processes leading to the final state $e^{-} e^{-} \rightarrow \mu^{-} e^{-}+\mathbb{E}$ are single $W^{-}$production (cf. B2), charged slepton-pair production (cf. B5), and single chargino production (cf. B7). The SM background $e^{-} \mu^{-} \nu_{e} \bar{\nu}_{\mu}$ originating mainly from single $W^{-}$production is estimated in Tab. प Including the cuts (i)-(iii) explained in the preceding subsection. As can be seen, the cross-sections are bigger and the cuts are less efficient than in the $e^{+} e^{-}$case. Moreover, contrary to what we have found for $e^{+} e^{-}$collisions, the situation at $\sqrt{s}=800 \mathrm{GeV}$ is somewhat worse than at $500 \mathrm{GeV}$ due to the increase of single $W^{-}$production with energy. The total cross-sections for the MSSM background processes are summarized in Tab. [5 In all four scenarios the MSSM background to $\mu^{-} e^{-}+\not E$ is below $1 \mathrm{fb}$, while the background to $\tau^{-} e^{-}+\not E$ in scenarios B and I is of the order of $100 \mathrm{fb}$. This is similar to our findings for $e^{+} e^{-}$collisions. Despite the larger background, the signal to background ratio tends to be slightly more favorable in $e^{-} e^{-}$collisions than in $e^{+} e^{-}$. For example, for scenario $\mathrm{C}$ and $\sqrt{s}=800 \mathrm{GeV}$ one finds roughly $S / \sqrt{B}\left(e^{-} e^{-}\right) \approx 2 S / \sqrt{B}\left(e^{+} e^{-}\right)$, assuming an integrated luminosity of 250 and $1000 \mathrm{fb}^{-1}$, respectively.

\section{Conclusions}

We have studied the lepton flavor-violating processes $e^{ \pm} e^{-} \rightarrow \sum_{i, j} \tilde{l}_{j}^{ \pm} \tilde{l}_{i}^{-} \rightarrow l_{\beta}^{ \pm} l_{\alpha}^{-} \tilde{\chi}_{b}^{0} \tilde{\chi}_{a}^{0}$ at a future linear $e^{+} e^{-}$collider. As a theoretical framework we have chosen mSUGRA benchmark scenarios furnished with the seesaw mechanism assuming a Majorana mass scale in the range $10^{11} \mathrm{GeV} \leq M_{R} \leq 10^{15} \mathrm{GeV}$. Our analysis shows that for center-of-mass energies of 500-800 GeV, the signal cross-sections may be as large as 1-10 fb. The detailed predic- 


\begin{tabular}{|c|c|c|c|c|c|c|c|}
\hline Final state & Production & \multicolumn{2}{|c|}{ B } & $\mathrm{C}$ & \multicolumn{2}{|c|}{$\mathrm{G}$} & $\mathrm{I}$ \\
\hline$e^{-} \mu^{-}+\not$ & & 500 & 800 & 800 & 500 & 800 & 800 \\
\hline & $\tilde{e}_{i}^{-} \tilde{e}_{i}^{-}$ & 0.01 & 0.01 & 0 & 0.01 & 0.2 & 0.4 \\
\hline & $e^{-} \tilde{\nu}_{e} \tilde{\chi}_{1}^{-}$ & 0 & 0.01 & 0.4 & - & 0.1 & 0 \\
\hline & Sum & 0.01 & 0.02 & 0.4 & 0.01 & 0.3 & 0.4 \\
\hline \hline$e^{-} \tau^{-}+\not E$ & & 500 & 800 & 800 & 500 & 800 & 800 \\
\hline & $\tilde{e}_{i}^{-} \tilde{e}_{i}^{-}$ & 140.8 & 102.3 & 0 & 0.2 & 8.1 & 60.0 \\
\hline & $e^{-} \tilde{\nu}_{e} \tilde{\chi}_{1}^{-}$ & 0 & 0.01 & 0.4 & - & 0.07 & 0 \\
\hline & Sum & 140.8 & 102.3 & 0.4 & 0.2 & 8.2 & 60.0 \\
\hline
\end{tabular}

Table 5: Cross-sections in fb for MSSM background processes in $e^{-} e^{-}$collisions at $\sqrt{s}=$ $500 \mathrm{GeV}$ and $800 \mathrm{GeV}$ as in Tab. 3 .

tions depend strongly on the SUSY parameters and on the neutrino masses and mixing. Consequently, they are also strongly affected by uncertainties in the neutrino data.

On the other hand, the correlations between the high-energy cross-sections and the branching ratios for the corresponding rare radiative decays are expected to be less influenced by experimental errors in neutrino parameters. We have therefore investigated these correlations very thoroughly and find that they are the stronger, the smaller the branching ratios for the radiative decays, that is the lower $M_{R}$. At $M_{R}$ smaller than $10^{13} \mathrm{GeV}$ the neutrino uncertainties play no role at all. The present bounds on $\mu \rightarrow e \gamma$ and $\tau \rightarrow \mu \gamma$ still allow sizable signals at a LC. If $\mu \rightarrow e \gamma$ will not be observed at the new PSI experiment, the cross-section for $e^{+} e^{-} \rightarrow \sum_{i, j} \tilde{l}_{j}^{+} \tilde{l}_{i}^{-} \rightarrow \mu^{+} e^{-}+2 \tilde{\chi}_{1}^{0}$ is predicted not to exceed $0.02 \mathrm{fb}$ at $\sqrt{s}=500 \mathrm{GeV}$. On the other hand, $\operatorname{Br}(\tau \rightarrow \mu \gamma)<10^{-9}$ alone would still be compatible with a cross-section of order $1 \mathrm{fb}$ for $e^{+} e^{-} \rightarrow \sum_{i, j} \tilde{l}_{j}^{+} \tilde{l}_{i}^{-} \rightarrow \tau^{+} \mu^{-}+2 \tilde{\chi}_{1}^{0}$, whereas $\operatorname{Br}(\mu \rightarrow e \gamma)<10^{-14}$ would be in conflict with such a large cross-section.

Finally, we have estimated the SM and MSSM background. With only a beam pipe cut of 10 degrees included, the SM background at 500 to $800 \mathrm{GeV}$ amounts to 80 to $100 \mathrm{fb}$ in the $\mu^{+} e^{-}$channel and to 20 to $60 \mathrm{fb}$ in the $\tau^{+} \mu^{-}$channel. The corresponding MSSM background varies between 0.01 and $10 \mathrm{fb}$ depending on the SUSY scenario, the final state and the beam energy. An exceptionally large MSSM background of 50 to $100 \mathrm{fb}$ is found in scenarios B and I in the channel $\tau^{+} e^{-}$which also contributes to the $\mu^{+} e^{-}$channel through leptonic $\tau$ decay. Since one can assume that the relevant sparticle spectra are known at the time such experiments can be done, it appears possible to sufficiently suppress the 
MSSM background by carefully designed cuts on decay energies, missing energy, angular distributions and other quantities. However, conclusive feasibility studies require a detailed Monte Carlo simulation.

The overall discovery potential for LFV at a LC is slightly increased by performing searches in $e^{-} e^{-}$collisions in addition to $e^{+} e^{-}$collisions.

\section{Acknowledgements}

We thank W. Porod for useful discussions. This work was supported by the Bundesministerium für Bildung und Forschung (BMBF, Bonn, Germany) under the contract number 05HT1WWA2 and (FD) by Spanish grant BFM2002-00345, by the European Commission RTN grant HPRN-CT-2000-00148 and European Commission Research Training Site grant HPMT-CT-2000-00124.

\section{References}

[1] Q. R. Ahmad et al. [SNO Collaboration], Phys. Rev. Lett. 87, 071301 (2001) arXiv:nucl-ex/0106015.

[2] Y. Fukuda et al. [Super-Kamiokande Collaboration], Phys. Rev. Lett. 81, 1562 (1998) arXiv:hep-ex/9807003.

[3] N. V. Krasnikov, Phys. Lett. B 388, 783 (1996) arXiv:hep-ph/9511464; N. ArkaniHamed, H. C. Cheng, J. L. Feng and L. J. Hall, Phys. Rev. Lett. 77, 1937 (1996) arXiv:hep-ph/9603431; N. Arkani-Hamed, J. L. Feng, L. J. Hall and H. C. Cheng, Nucl. Phys. B 505, 3 (1997) arXiv:hep-ph/9704205; H. C. Cheng, arXiv:hep-ph/9712427; M. Hirouchi and M. Tanaka, Phys. Rev. D 58, 032004 (1998) arXiv:hep-ph/9712532; J. L. Feng, Int. J. Mod. Phys. A 13, 2319 (1998) arXiv:hep-ph/9803319; J. Hisano, M. M. Nojiri, Y. Shimizu and M. Tanaka, Phys. Rev. D 60, 055008 (1999) arXiv:hep-ph/9808410; D. Nomura, Phys. Rev. D 64, 075001 (2001) arXiv:hep-ph/0004256; M. Dine, Y. Grossman and S. Thomas, eConf C010630, P332 (2001) [Int. J. Mod. Phys. A 18, 2757 (2003)] arXiv:hep-ph/0111154; M. Cannoni, S. Kolb and O. Panella, Phys. Rev. D 68, 096002 (2003) arXiv:hep-ph/0306170; J. Cao, Z. Xiong and J. M. Yang, arXiv:hep-ph/0307126. 
[4] M. Guchait, J. Kalinowski and P. Roy, Eur. Phys. J. C 21, 163 (2001) arXiv:hep-ph/0103161.

[5] W. Porod and W. Majerotto, Phys. Rev. D 66, 015003 (2002) arXiv:hep-ph/0201284.

[6] M. Gell-Mann, P. Ramond and R. Slansky, Proceedings of the Supergravity Stony Brook Workshop, New York 1979, eds. P. Van Nieuwenhuizen and D. Freedman; T. Yanagida, Proceedings of the Workshop on Unified Theories and Baryon Number in the Universe, Tsukuba, Japan 1979, eds. A. Sawada and A. Sugamoto; R. N. Mohapatra and G. Senjanovic, Phys. Rev. Lett. 44, 912 (1980), erratum ibid Phys. Rev. D 23, 165 (1993).

[7] F. Deppisch, H. Päs, A. Redelbach, R. Rückl and Y. Shimizu, Eur. Phys. J. C 28, 365 (2003) arXiv:hep-ph/0206122.

[8] M. Battaglia et al., Eur. Phys. J. C 22, 535 (2001) arXiv:hep-ph/0106204.

[9] J. A. Casas and A. Ibarra, Nucl. Phys. B 618, 171 (2001) arXiv:hep-ph/0103065.

[10] W. de Boer, Prog. Part. Nucl. Phys. 33, 201 (1994) arXiv:hep-ph/9402266.

[11] S. T. Petcov, S. Profumo, Y. Takanishi and C. E. Yaguna, arXiv:hep-ph/0306195.

[12] F. Deppisch, A. Redelbach and R. Rückl, in preparation.

[13] J. Hisano and D. Nomura, Phys. Rev. D 59, 116005 (1999) arXiv:hep-ph/9810479; J. R. Ellis, J. Hisano, M. Raidal and Y. Shimizu, Phys. Lett. B 528, 86 (2002) arXiv:hep-ph/0111324.

[14] F. Deppisch, H. Päs, A. Redelbach and R. Rückl, in preparation.

[15] H. E. Haber and G. L. Kane, Phys. Rept. 117, 75 (1985).

[16] F. Deppisch, Diploma Thesis, Würzburg 2000, available at ftp://ftp.physik.uni-wuerzburg.de/pub/diplom/deppisch.ps.gz.

[17] A. Redelbach, Diploma Thesis, Würzburg 2000, available at ftp://ftp.physik.uni-wuerzburg.de/pub/diplom/redelbach.ps.gz.

[18] M. C. Gonzalez-Garcia, M. Maltoni, C. Pena-Garay and J. W. Valle, Phys. Rev. D 63, 033005 (2001) arXiv:hep-ph/0009350.

[19] M. Maltoni, T. Schwetz, M. A. Tortola and J. W. F. Valle, arXiv:hep-ph/0309130. 
[20] K. Hagiwara et al. [Particle Data Group Collaboration], Phys. Rev. D 66, 010001 (2002).

[21] L. M. Barkov et al., Research Proposal R-99-05.1 for an experiment at PSI (1999).

[22] K. Inami, T. Hokuue and T. Ohshima [BELLE Collaboration], eConf C0209101, TU11 (2002) arXiv:hep-ex/0210036.

[23] L. Serin and R. Stroynowski, ATLAS Internal Note (1997); T. Ohshima, talk at the 3rd Workshop on Neutrino Oscillations and their Origin (NOON2001), 2001, ICRR, Univ. of Tokyo, Kashiwa, Japan; D. Denegri, private communication.

[24] A. Pukhov et al., arXiv:hep-ph/9908288.

[25] R. Becker and C. Vander Velde, IIHE-93-08, 457 (1993), in Proceedings of the Workshop on $e^{+} e^{-}$Collisions at 500 GeV: The Physics Potential, Part C, ed. P.M. Zerwas, DESY93-123C, 457 (1993).

[26] J. Gluza and M. Zralek, Phys. Lett. B 362, 148 (1995) arXiv:hep-ph/9507269. 

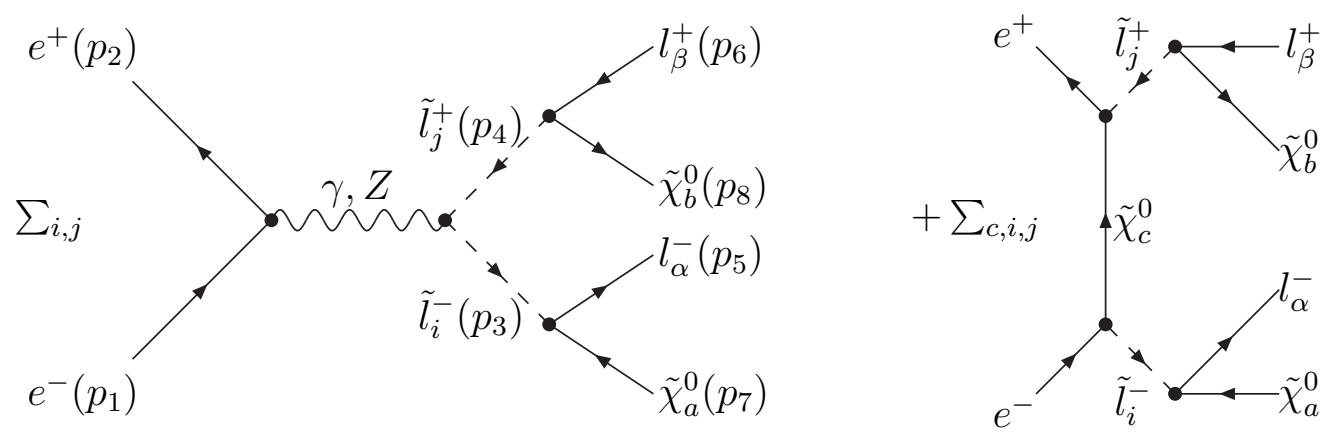

Figure 1: Feynman diagrams for $e^{+} e^{-} \rightarrow \tilde{l}_{j}^{+} \tilde{l}_{i}^{-} \rightarrow l_{\beta}^{+} l_{\alpha}^{-} \tilde{\chi}_{b}^{0} \tilde{\chi}_{a}^{0}$. The arrows on scalar lines refer to the lepton number flow.

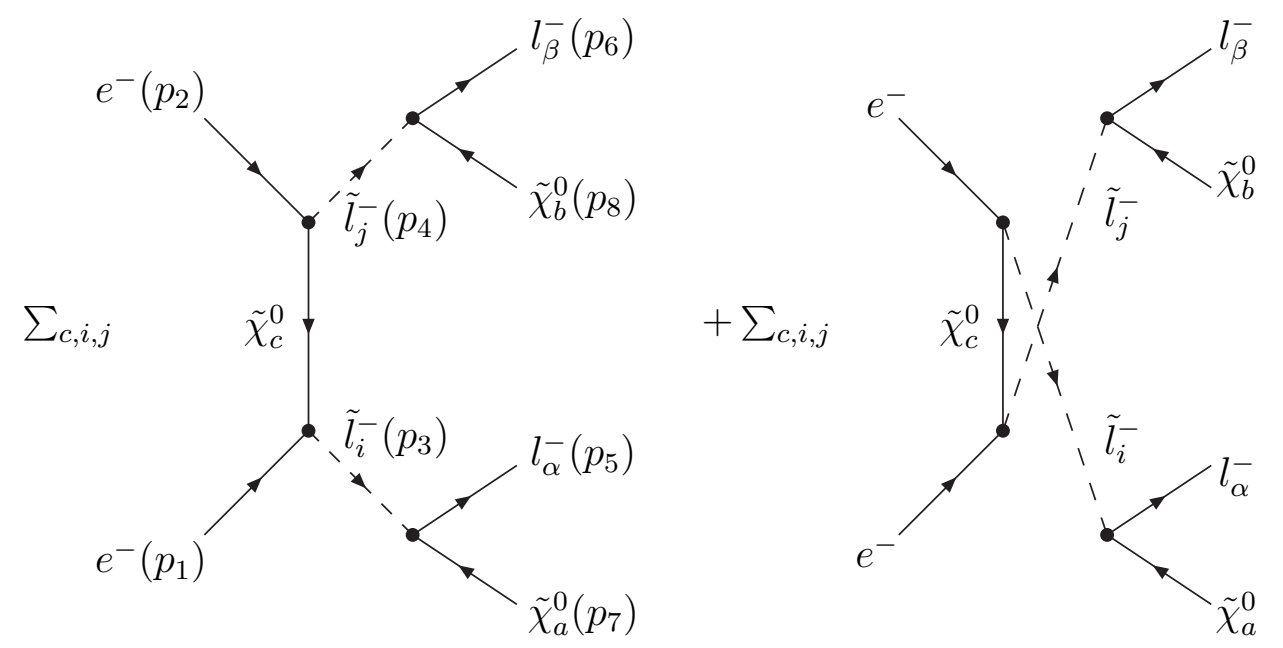

Figure 2: Feynman diagrams for $e^{-} e^{-} \rightarrow \tilde{l}_{j}^{-} \tilde{l}_{i}^{-} \rightarrow l_{\beta}^{-} l_{\alpha}^{-} \tilde{\chi}_{b}^{0} \tilde{\chi}_{a}^{0}$. The arrows on scalar lines refer to the lepton number flow. 


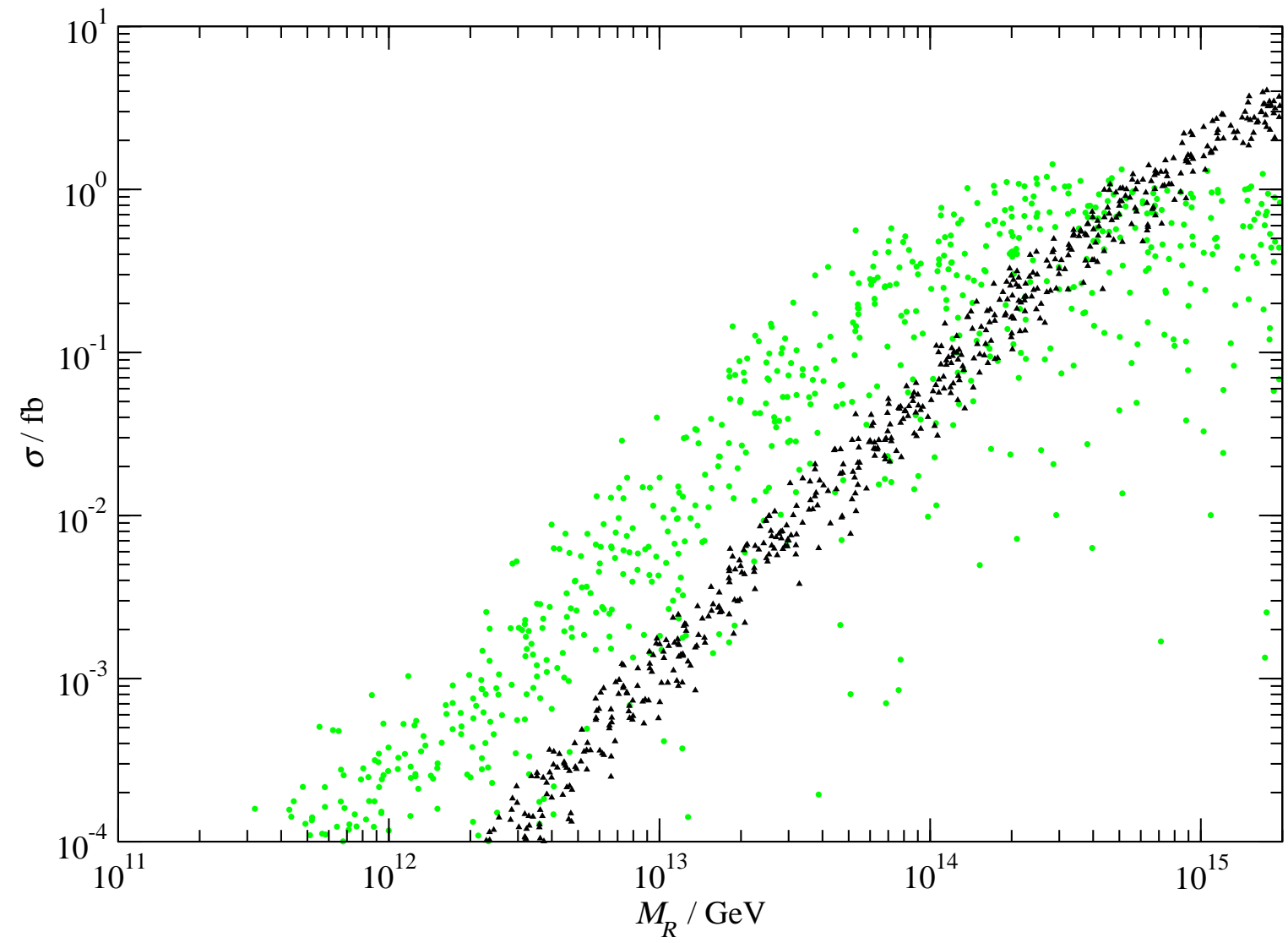

Figure 3: Cross-sections at $\sqrt{s}=500 \mathrm{GeV}$ for $e^{+} e^{-} \rightarrow \mu^{+} e^{-}+2 \tilde{\chi}_{1}^{0}$ (circles) and $e^{+} e^{-} \rightarrow$ $\tau^{+} \mu^{-}+2 \tilde{\chi}_{1}^{0}$ (triangles) in scenario B for the case of very light neutrinos. 


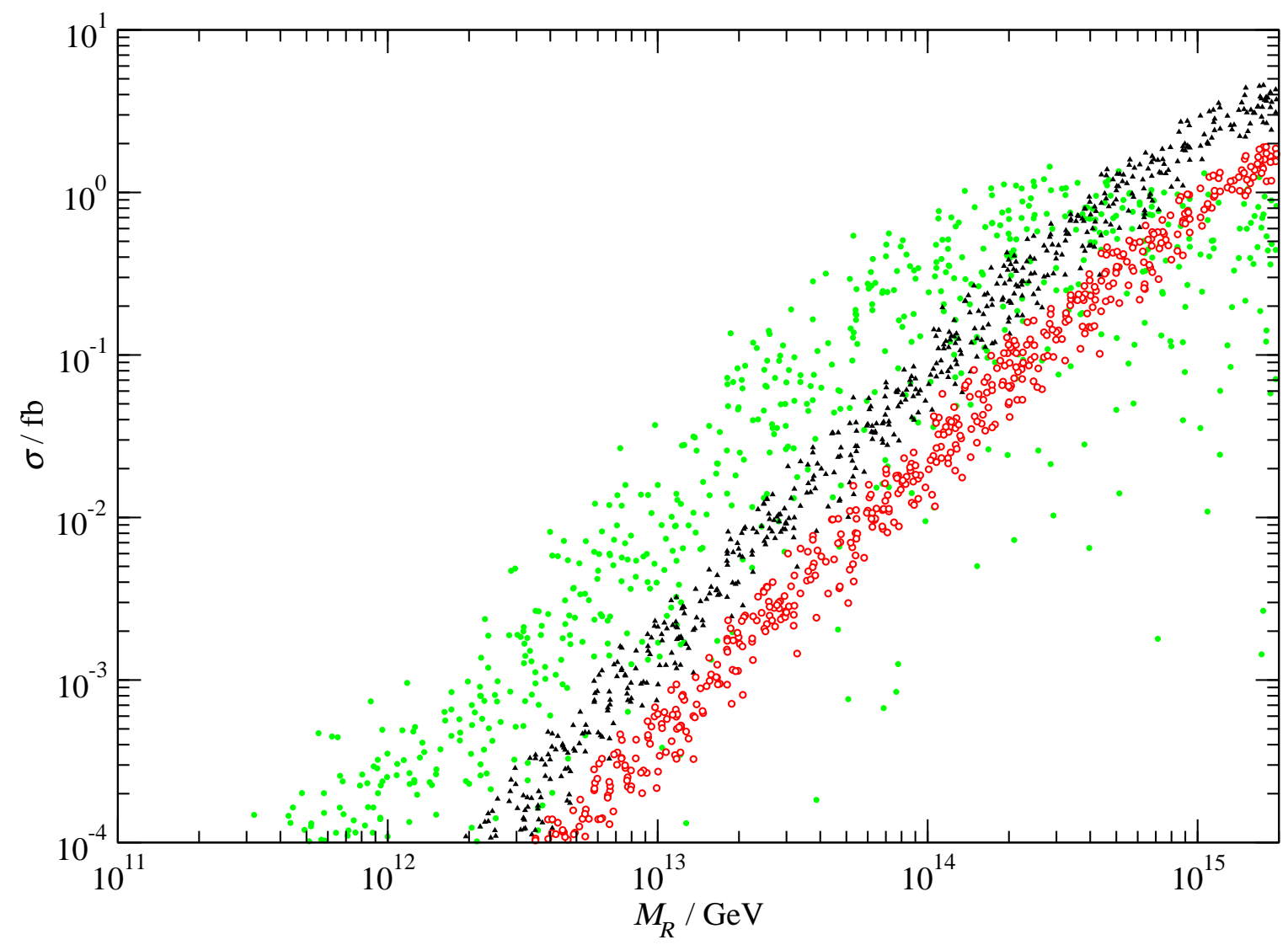

Figure 4: Cross-sections at $\sqrt{s}=800 \mathrm{GeV}$ for $e^{+} e^{-} \rightarrow \mu^{+} e^{-}+2 \tilde{\chi}_{1}^{0}$ in scenario B (circles) and $e^{+} e^{-} \rightarrow \tau^{+} \mu^{-}+2 \tilde{\chi}_{1}^{0}$ in scenario $\mathrm{B}$ (triangles) and I (open circles) for the case of very light neutrinos. 


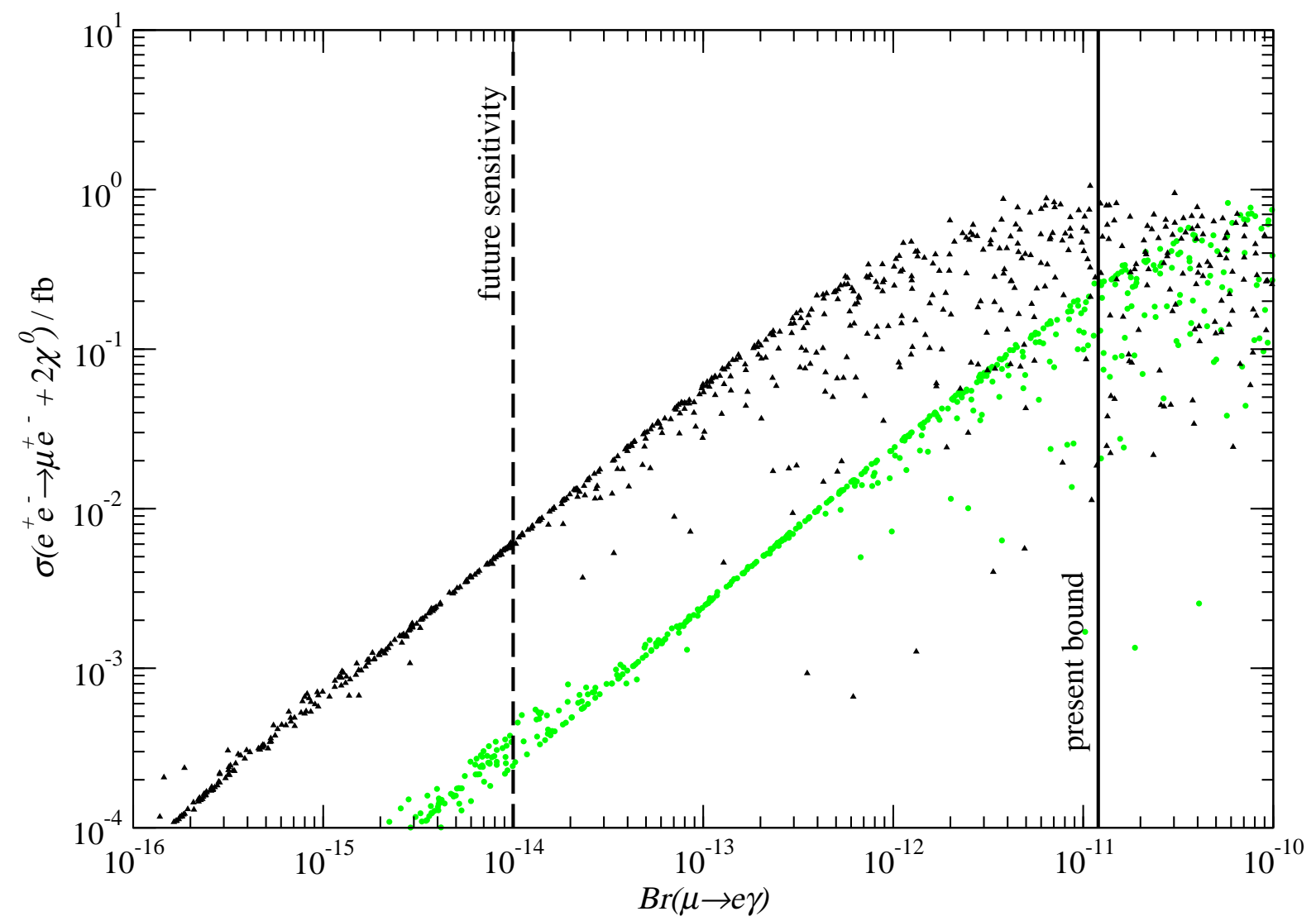

Figure 5: Correlation of $\sigma\left(e^{+} e^{-} \rightarrow \mu^{+} e^{-}+2 \tilde{\chi}_{1}^{0}\right)$ at $\sqrt{s}=500 \mathrm{GeV}$ with $\operatorname{Br}(\mu \rightarrow e \gamma)$ in scenario $\mathrm{C}$ (triangles) and $\mathrm{B}$ (circles) for the case of very light neutrinos. 


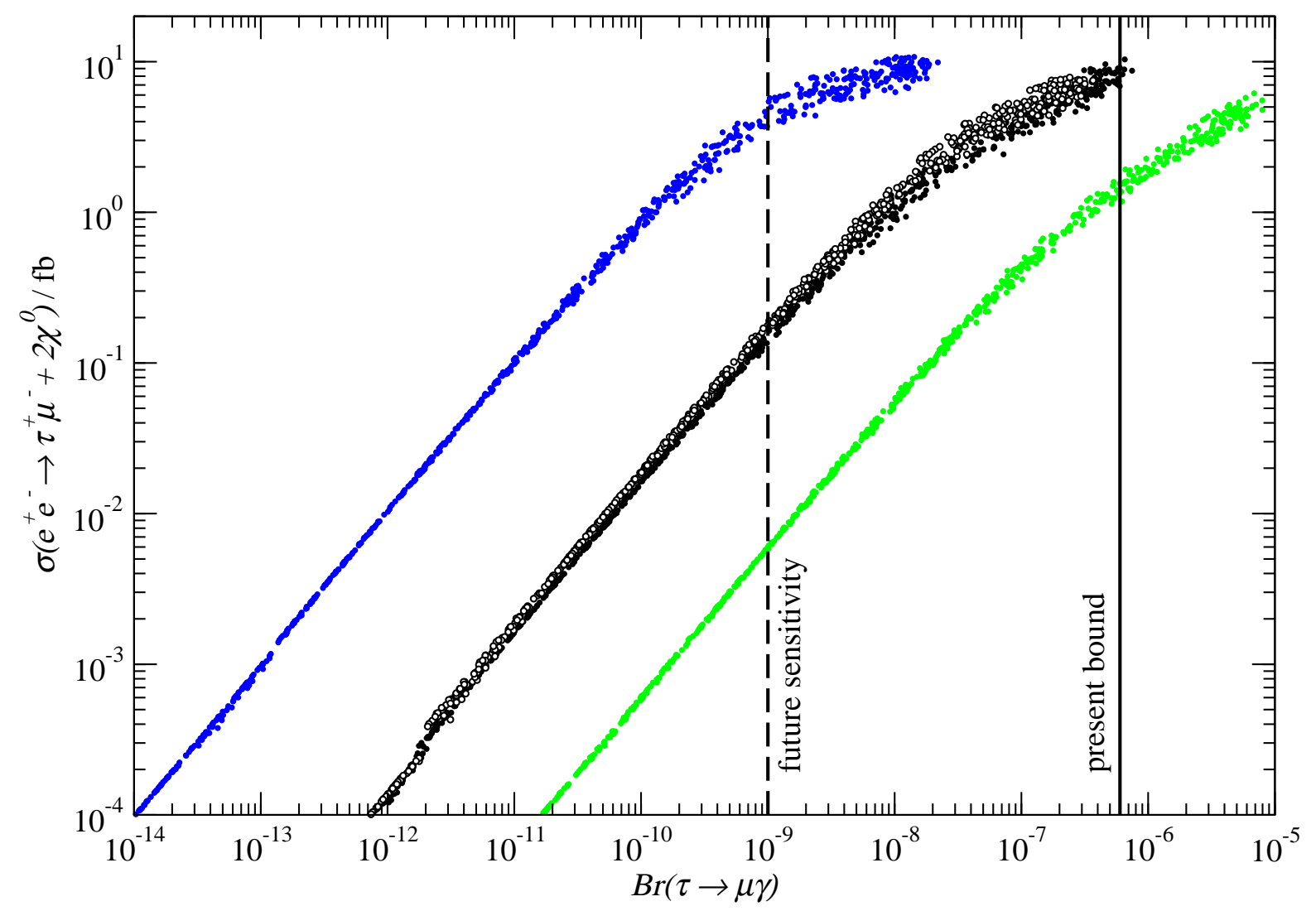

Figure 6: Correlation of $\sigma\left(e^{+} e^{-} \rightarrow \tau^{+} \mu^{-}+2 \tilde{\chi}_{1}^{0}\right)$ at $\sqrt{s}=800 \mathrm{GeV}$ with $\operatorname{Br}(\tau \rightarrow \mu \gamma)$ in scenario (from left to right) C, G (open circles), B and I for the case of very light neutrinos. 


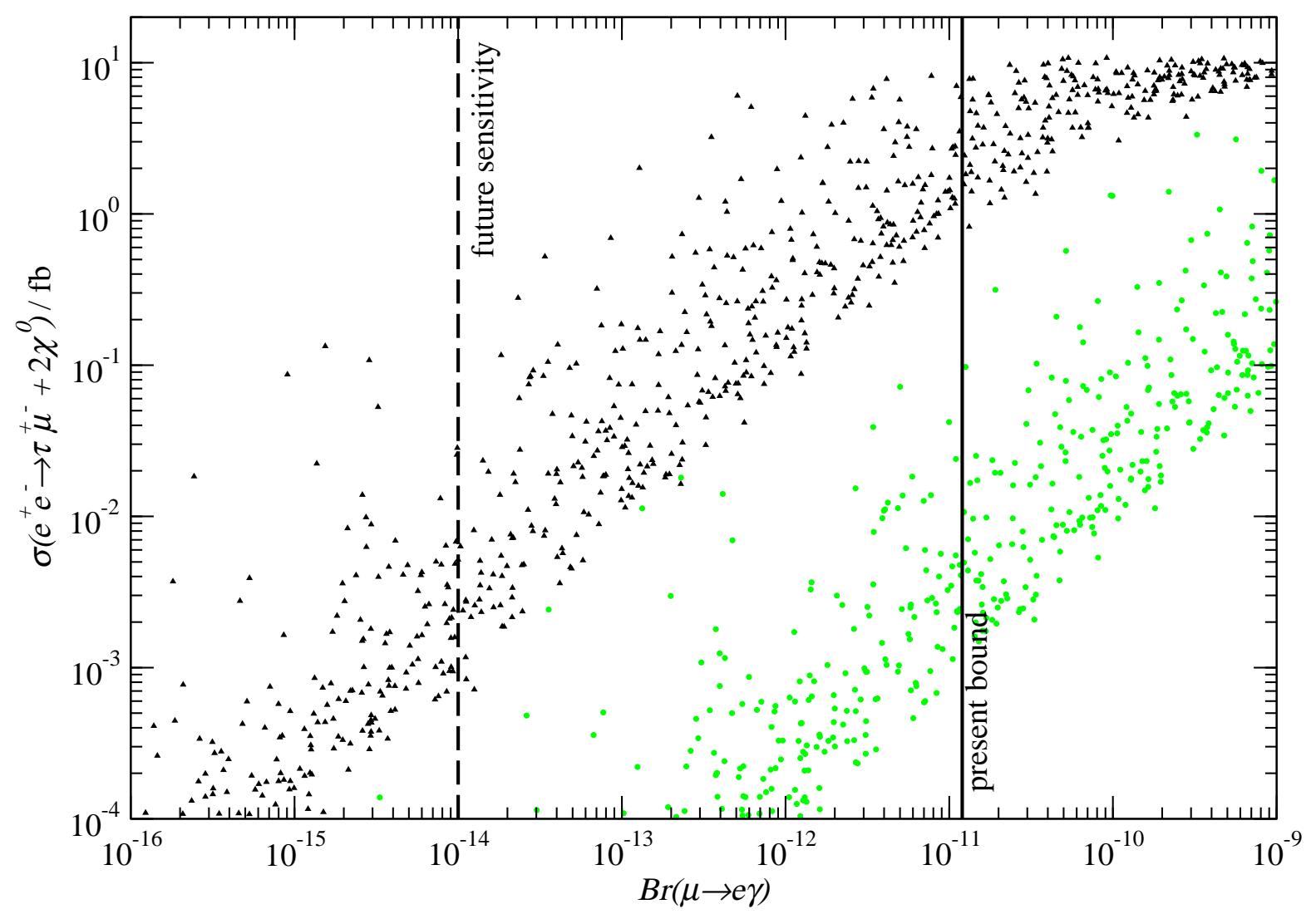

Figure 7: Correlation of $\sigma\left(e^{+} e^{-} \rightarrow \tau^{+} \mu^{-}+2 \tilde{\chi}_{1}^{0}\right)$ at $\sqrt{s}=800 \mathrm{GeV}$ with $\operatorname{Br}(\mu \rightarrow e \gamma)$ in scenario $\mathrm{C}$ (triangles) and I (circles) for the case of very light neutrinos. 


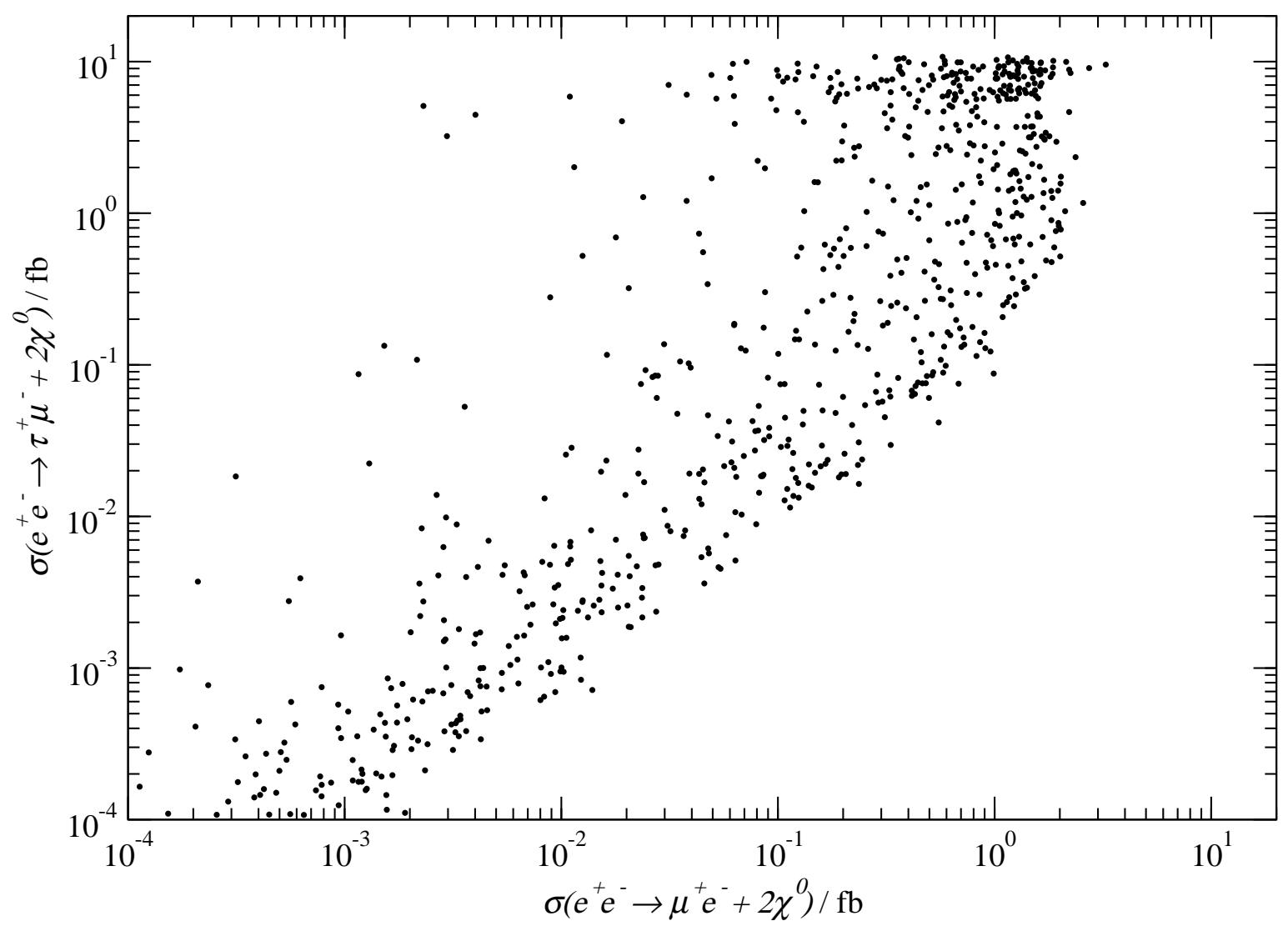

Figure 8: Correlation of $\sigma\left(e^{+} e^{-} \rightarrow \tau^{+} \mu^{-}+2 \tilde{\chi}_{1}^{0}\right)$ with $\sigma\left(e^{+} e^{-} \rightarrow \mu^{+} e^{-}+2 \tilde{\chi}_{1}^{0}\right)$ at $\sqrt{s}=800$ $\mathrm{GeV}$ in scenario $\mathrm{C}$ for the case of very light neutrinos. 


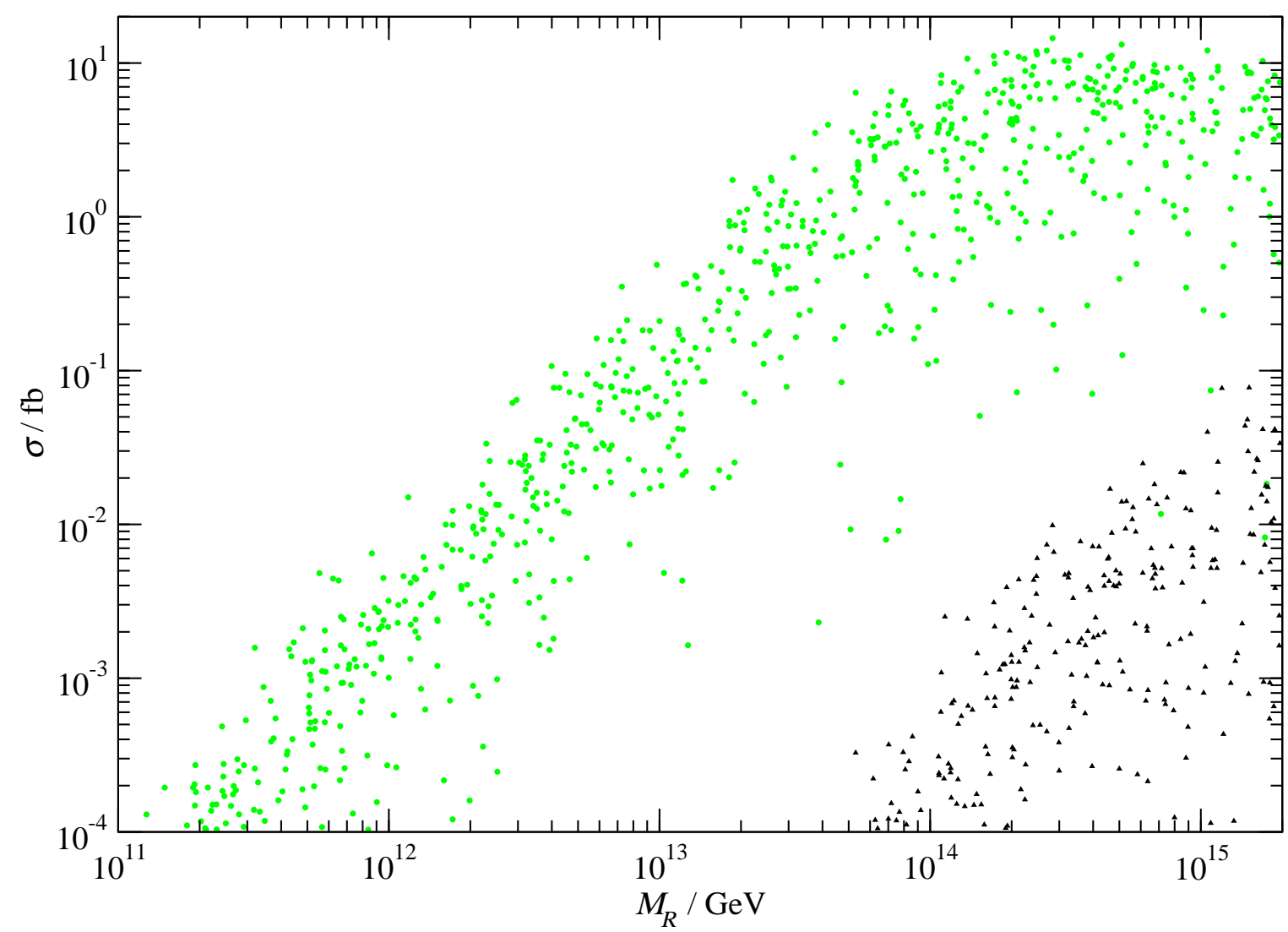

Figure 9: Cross-sections at $\sqrt{s}=800 \mathrm{GeV}$ for $e^{-} e^{-} \rightarrow \mu^{-} e^{-}+2 \tilde{\chi}_{1}^{0}$ (circles) and $e^{-} e^{-} \rightarrow$ $\tau^{-} \mu^{-}+2 \tilde{\chi}_{1}^{0}$ (triangles) in scenario $\mathrm{C}$ for the case of very light neutrinos. 


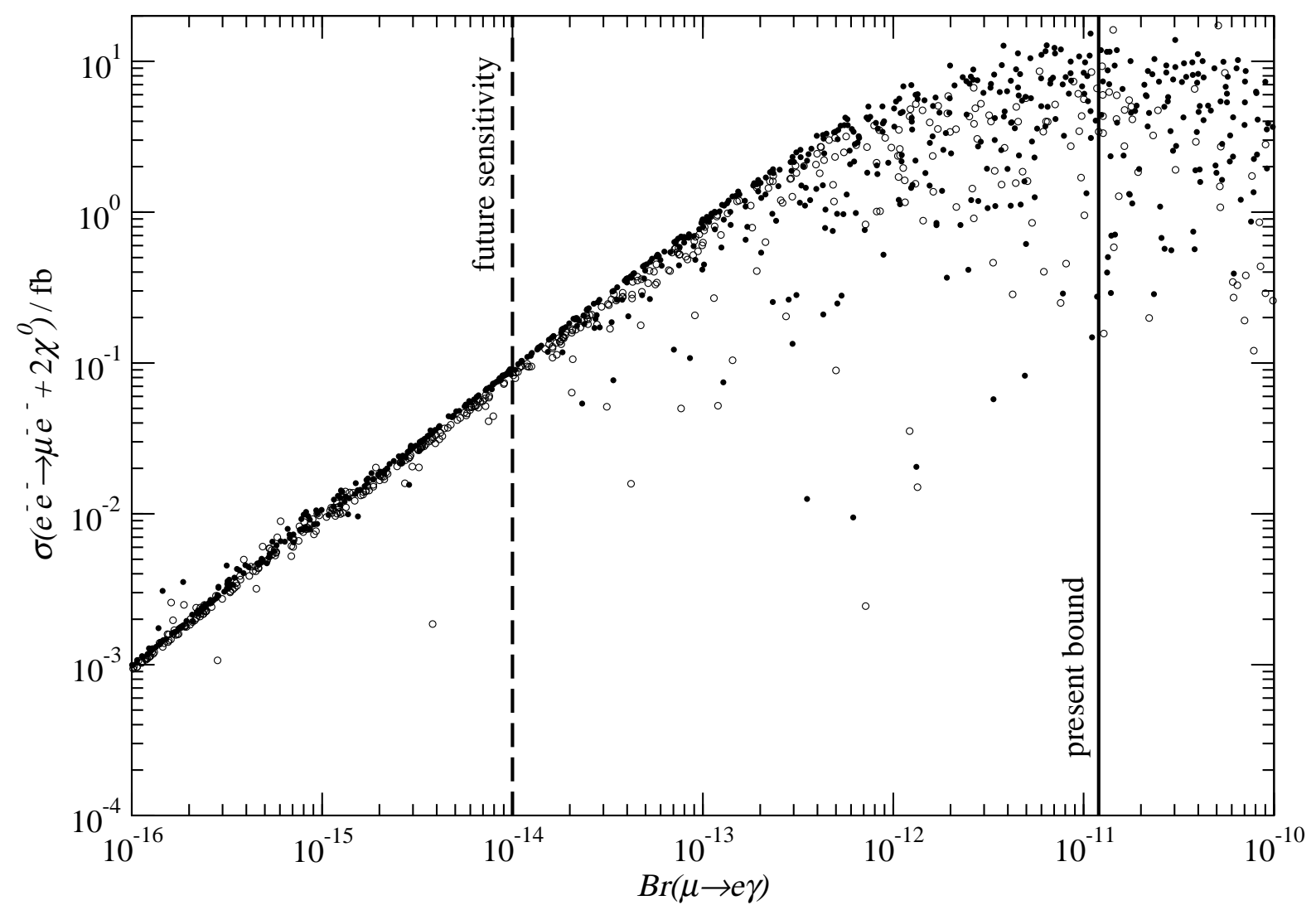

Figure 10: Correlation of $\sigma\left(e^{-} e^{-} \rightarrow \mu^{-} e^{-}+2 \tilde{\chi}_{1}^{0}\right)$ at $\sqrt{s}=800 \mathrm{GeV}$ with $\operatorname{Br}(\mu \rightarrow e \gamma)$ in scenario $\mathrm{C}$ for the case of very light neutrinos (closed circles) and degenerate heavier neutrinos (open circles). 